\title{
THE RADON-NIKODÝM THEOREM FOR FINITE RINGS OF OPERATORS
}

\author{
BY \\ H. A. DYE
}

1. Introduction. Of the reasons for the advanced and elegant development of the theory of abelian operator algebras on a hilbert space, none perhaps is more decisive than the essential measure-theoretic character of such algebras. Particularly in the multiplicity theory, in which the invariants of weakly closed rings turn out to be purely measure-theoretic objects, the analogies and interplay between measure theory and spectral theory provide the most critical methods. To a remarkable degree, this same connection subsists between the general theory of weakly closed operator algebras and measure theory, or properly speaking, "noncommutative measure theory." The study of rings of operators-fostered in the main by J. von Neumann, and advanced in the more recent works of J. Dixmier, I. Kaplansky, I. Segal, R. Godement, and many others-as we know, hinges on noncommutative notions of dimension and invariant integrals, and gives rise to natural and fruitful notions of $L_{2}$ and $L_{1}$ systems. In the last analysis, the multiplicity theory for general rings will probably derive from an extension of measure theory to this noncommutative situation.

The present memoir is devoted to a natural step in this general integration theory: our central objective is the generalization of the classical RadonNikodým theorem (for finite measure spaces) to any finite ring of operators. Briefly put, our formulation of this theorem runs as follows. We consider any $W^{*}$-algebra $M$ which is $\sigma$-finite, in the sense that any collection of mutually disjoint projections in $M$ is at most countable, and finite, in the sense that no projection in $M$ less than the identity is equivalent $\bmod M$ to the identity; this class of rings contains all factors of type $I_{n}$ and $I_{1}$, as well as all abelian rings whose commutants have cyclic vectors. In the role of measures (or rather, integrals) we consider positive functionals (=states) on $M$ which are countably additive ( $=\mathrm{CA}$ ) on sequences of mutually orthogonal projections in $M$. Between two such states $\sigma$ and $\rho$ a notion of absolute continuity is defined by setting $\sigma<\rho(M)$ in event $\rho(P)=0$ implies $\sigma(P)=0$, for each projection $P$ in $M$. We then associate with each CA state $\rho$ of $M$ a hilbert space $L_{2}(M, \rho)$, whose elements are closed densely-defined operators belonging to $M$, and prove (in $\$ 5$ ) the key theorem: if $\sigma<\rho(M)$, then $\sigma(A)=\rho\left(T^{*} A T\right)$, for some $T$ in $L_{2}(M, \rho)$.

In attacking the main problem, we begin in $\$ 3$ by considering the case in which the CA state $\rho$ is canonical (that is, of the form $\rho(A)=(A x, x)$ ). By

Received by the editors June 22, 1951. 
appealing to techniques suggested by the Murray-von Neumann "BTtheorem" [8, Lemma 9.2.1], we show for this case that the finiteness of all cyclic projections $E_{\left[M^{\prime} x\right]}$ in $M$ is both necessary and sufficient for the unrestricted validity of the Radon-Nikodým theorem. Btfore passing to the global case, we ascribe a notion of "length" to the identity $I$ in a $\sigma$-finite finite $W^{*}$-algebra $M$, this being the smallest (finite or infinite) number of cyclic projections in $M$ whose union is $I$, and prove in $\$ 4$ that any $\sigma$-finite finite $W^{*}$-algebra is invariantly expressible as a central direct sum of finite $W^{*}$-algebras uniformly of finite length. With these facts in hand, we show that any CA state of a $\sigma$-finite finite $W^{*}$-algebra has the form $\sum_{i=1}^{n}\left(A x_{i}, x_{i}\right)$, where $n(=\infty, 1,2, \cdots)$ is the length of the identity in $M$, and use this concrete representation in conjunction with the remarkable calculus of unbounded operators so characteristic of finite rings to construct $L_{2}(M, \rho)$ and prove the Radon-Nikodým theorem.

The existence of unitarily invariant CA states on any finite ring (a fact established by Dixmier in [3]) plays an essential role in our proof of the global theorem, and in event the state $\rho$ is unitarily invariant, the system $L_{2}(M, \rho)$ enjoys some remarkable properties (cited below) and the RadonNikodým theorem itself assumes a sharper form. There is a distinct advantage in considering all CA states, however; by virtue of this generality, we obtain easily a number of topological by-products bearing out the strong topological restrictions imposed by the algebraic notion of finiteness. It is shown, for example, that any (algebraic) ${ }^{*}$-isomorphism between finite $W^{*}$-algebras is bicontinuous in the strongest topology, and that a ${ }^{*}$-isomorphic image of a $\sigma$-finite finite $W^{*}$-algebra in a $\sigma$-finite ring is always weakly closed.

In the concluding section, by analyzing the $L_{2}$-system of a finite ring formed with respect to a unitarily invariant state, we show that any $\sigma$-finite finite $W^{*}$-algebra is ${ }^{*}$-isomorphic to the bounded algebra in a finite $H$-system (a structure defined by Ambrose in [1] which generalizes in the finite case the $L_{2}$-system of a discrete group) and, furthermore, that two $\sigma$-finite finite $W^{*}$-algebras are ${ }^{*}$-isomorphic if and only if their associated $H$-systems are unitarily equivalent. Besides providing a rich prototype model for $\sigma$-finite finite $W^{*}$-algebras-much akin to the algebra of multiplications by bounded measurable functions on $L_{2}$ of a finite measure space-this theorem shows that the classification of $\sigma$-finite finite $W^{*}$-algebras up to ${ }^{*}$-isomorphisms is tantamount to the classification of finite $H$-systems up to unitary equivalence.

Our technical resources in this work are drawn heavily from the fundamental paper of Murray and von Neumann [8]. Of the main results in $\S \S 5$ and 6 , Theorems 4 and 5 were originally conjectured to the author by I. E. Segal, as were several of the corollaries by I. Kaplansky. For these sources, the author wishes to acknowledge his very deep gratitude.

2. Countably additive states. This section is devoted to an exposition of preliminary materials, centering on the concept of "countably additive 
states." The principal result (Theorem 1) is a local Radon-Nikodým theorem, which shows that each CA state of a $\sigma$-finite $W^{*}$-algebra has a local canonical form $(A x, x)$. This leads to a simple proof of the von Neumann trace formula [9, Theorem 3], and to a proof that any CA state of a $\sigma$-finite $W^{*}$-algebra is strong sequentially continuous.

Notation 2.1. Let $\mathfrak{S}$ be a fixed complex hilbert space. By a ring of operators (or simply a ring) $M$ on $\mathfrak{S}$ we mean a self-adjoint algebra of operators on $\mathfrak{S}$ closed in the weak neighborhood topology. Following Segal, we call a ring $M$ a $W^{*}$-algebra if $M$ contains the identity operator. Of the fundamental properties of $W^{*}$-algebras, we shall make most use of the following two: if $M^{\prime}$ (= the "commutant of $M$ ") denotes the collection of all bounded linear operators on $\mathfrak{S}$ which commute with all elements of $M$, then $\left(M^{\prime}\right)^{\prime}=M$; and if $M_{P}$ denotes the collection of all projections in $M$, then $M$ is the smallest uniformly closed *-algebra on $\mathfrak{S}$ containing $M_{P}$, and, furthermore, $M_{P}$ is a complete lattice under the natural (extrinsic) definitions of meet and join-namely, if $\mathfrak{M}$ and $\mathfrak{N}$ are subspaces in $\mathfrak{L}$, if $E_{\mathfrak{M}}$ and $E_{\mathfrak{R}}$ denote the projections on these subspaces, and if $\mathfrak{M} \oplus \mathfrak{N}$ and $\mathfrak{M} \cap \mathfrak{N}$ denote respectively the smallest closed subspace of $\mathfrak{S}$ containing $\mathfrak{M}$ and $\mathfrak{R}$ and the intersection of

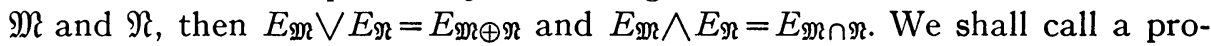
jection $P$ in $M \sigma$-finite if any family of mutually orthogonal projections in $M$ and less than $P$ is at most countable; in particular, $M$ itself is $\sigma$-finite if the identity in $M$ is $\sigma$-finite. (Segal in [15] uses the term "countably decomposable" for this concept.) If $x$ is any vector in $\mathfrak{S},[M x]$ will denote the closure of the manifold $[A x \mid A$ in $M]$. Notice that $E_{\left[M^{\prime} x\right]} \in M_{P}$ and that $E_{\left[M^{\prime} x\right]}$ is $\sigma$-finite.

In our work, a state $\rho$ of any uniformly closed self-adjoint algebra $M$ containing the identity operator $I\left(=C^{*}\right.$-algebra) will be any nonzero complex-valued linear functional on $M$ with the property that $\rho(A) \geqq 0$, for each non-negative self-adjoint (=SA) $A$ in $M$. We call a state normalized if $\rho(I)=1$ (this in apposition to the usual convention, by which all states are normalized). Certain elementary properties of states of $C^{*}$-algebras (with identity) will be used in the sequel without due remark-for example, the fact that states are continuous in the uniform topology, the fact that they are real, in the sense that $\rho\left(A^{*}\right)=\bar{\rho}(A)$, for all $A$, and the fact that they satisfy the Schwarz inequality, $\left[\left.\rho\left(B^{*} A\right)\right|^{2} \leqq \rho\left(B^{*} B\right) \rho\left(A^{*} A\right)\right.$, for all $A$ and $B$. For a discussion of these facts, see Neumark [12, §6]. If $A \in M$ and if $\rho$ is a state of $M$, we denote by $\rho_{A}$ that state of $M$ defined by the equation $\rho_{A}(B)=\rho\left(A^{*} B A\right)$. And finally, by a canonical state $\omega_{x}$ of $M$, we mean a functional on $M$ of the form $\omega_{x}(A)=(A x, x)$, where $x$ is any nonzero vector in $\mathfrak{S}$.

Definition 2.1. A state $\rho$ of the $W^{*}$-algebra $M$ is called countably additive ( $=\mathrm{CA})$ in case $\rho\left(\sum P_{n}\right)=\sum \rho\left(P_{n}\right)$, for each sequence $\left\{P_{n}\right\}$ of mutually orthogonal projections in $M$.

Our discussions will be focused on CA states of $\sigma$-finite rings. In this sec- 
tion, at least, it would come to the same thing to discuss completely additive states of an arbitrary ring.

Lemмa 2.1. With each $C A$ state of a $\sigma$-finite $W^{*}$-algebra $M$ there is associated a unique projection $E_{\rho}$ with the following property: for each $Q$ in $M_{P}$, $\rho(Q)=0$ if and only if $Q\left(I-E_{\rho}\right)=Q$.

Proof. Let $N=\left[P\right.$ in $\left.M_{P} \mid \rho(P)=0\right]$. If $\left\{P_{s}\right\}_{s \in S}$ is a maximal set of mutually orthogonal nonzero projections in $N$, it follows by $\sigma$-finiteness and countable additivity that the projection $I-E_{\rho}=\sum_{8} P_{s}$ likewise belongs to $N$. Observe that, for any $Q$ in $N$, we have $Q \vee\left(I-E_{\rho}\right) \in N$; in fact, $Q$ $\bigvee\left(I-E_{\rho}\right)$ is the projection on the range of $Q+I-E_{\rho}$, and by the spectral theorem, if $\rho(A)=0$, for any non-negative SA $A$ in $M$, then $E_{\text {[Range A] }} \in N$. It follows that $\left(I-E_{\rho}\right)$ is maximal in $N$, and our conclusion is easily obtained.

Suggestively, we shall call the projection $E_{\rho}$ the carrier projection of the CA state $\rho$. Notice that, for any canonical state $\omega_{x}, E_{\omega_{x}}=E_{\left[M^{\prime} x\right]}$.

Before introducing the general notion of absolute continuity, we shall describe a much restrictive concept (called "subordination" by Neumark, who introduces this notion in $[12, \S 9]$ ) which will nonetheless play an important role.

Definition 2.2. Two states $\sigma$ and $\rho$ of the $C^{*}$-algebra $M$ are in the relation $\sigma \ll \rho(M)$ if, for each sequence $\left\{A_{n}\right\}$ in $M$ such that $\rho\left(A_{n}^{*} A_{n}\right) \rightarrow 0$, it follows also that $\sigma\left(A_{n}^{*} A_{n}\right) \rightarrow 0$.

As is easily seen, this is equivalent to the existence of a (positive) constant $K$ such that $K \rho-\sigma$ is a state.

Lemma 2.2. Let $\rho$ be a state of the $C^{*}$-algebra $M$. Then $\rho \ll \omega_{x}(M)$ if and only if $\rho=\omega_{T x}(M)$, for some $T$ in $M^{\prime}$.

Proof. It is plain that $\omega_{T, x} \ll \omega_{x}(M)$, for any $T$ in $M^{\prime}$. Conversely, given $\rho \ll \omega_{x}(M)$, define a bilinear form $[A x, B x]$ on the manifold $M x$ by setting $[A x, B x]=\rho\left(B^{*} A\right)$. The ascribed properties of $\rho$ show that $[A x, B x]$ is bounded, positive semidefinite, and hermitian bilinear. Such a bilinear form always has a representation $[A x, B x]=\left(A x, T_{0} B x\right)$, where $T_{0}$ is a bounded non-negative SA operator on $[M x]$. In addition, $\left(A x, T_{0} C B x\right)=\rho\left((C B)^{*} A\right)$ $=\rho\left(\left(B^{*}\right) C^{*} A\right)=\left(A x, C T_{0} B x\right)$, for all $A, B, C$ in $M$, so that $T_{0} C=C T_{0}$ on [Mx]. Therefore, $T_{0} E_{[M x]}$ is a non-negative SA operator in $M^{\prime}$, and by virtue of the spectral theorem, $T_{0} E_{[M x]}$ has a square root $T$ in $M^{\prime}$. This operator $T$ gives the desired representation, $\rho=\omega_{T x}(M)$.

Definition 2.3. Let $\sigma$ and $\rho$ be states of the $W^{*}$-algebra $M$. We shall say that $\sigma$ is absolutely continuous with respect to $\rho$, and write $\sigma<\rho(M)$, if $\rho(P)=0$ implies $\sigma(P)=0$, for each $P$ in $M_{P}$. If in addition $\rho<\sigma(M)$, we shall write $\rho \sim \sigma(M)$.

An alternative definition of $\sigma<\rho(M)$ for CA states is the following: $\rho\left(A_{n}^{*} A_{n}\right) \rightarrow 0$ implies $\sigma\left(A_{n}^{*} A_{-n}\right) \rightarrow 0$, for each uniformly bounded sequence $\left\{A_{n}\right\}$ 
in $M$. This fact is an easy consequence of Lemma 2.3 below.

Theorem 1. Let $\rho$ be a $C A$ state of the $\sigma$-finite $W^{*}$-algebra $M$, and let $E$ be any projection in $M, 0<E \leqq E_{\rho}$. Then there exists a projection $P$ in $M$, $0<P \leqq E$, and $a$ vector $x$ in $\mathfrak{S}$ such that $\rho_{P}=\omega_{x}(M)$.

Proof. Choose any vector $z$ such that $\rho(E) \leqq(E z, z)$. According to Lemma 2.2 , it will suffice to exhibit a $P$ in $M_{P}, 0<P \leqq E$, such that $\rho_{P} \ll \omega_{P z}(M)$. In turn, we assert, it suffices to exhibit a $P$ in $M_{P}, 0<P \leqq E$, such that $\rho(Q)$ $\leqq \omega_{z}(Q)$, for all $Q$ in $M_{P}$ satisfying $Q \leqq P$. In fact, this being true, it follows that $\rho_{P}(A) \leqq \omega_{P z}(A)$, for all non-negative SA $A$ in $M$-as one sees by expressing $P A P$ as a uniform limit of finite linear combinations $\sum \alpha_{i} Q_{i}$, with $\alpha_{i} \geqq 0, Q_{i} \in M_{P}$, and $Q_{i} \leqq P$, and applying the inequality to these linear combinations-and the relation $\rho_{P} \ll \omega_{P z}(M)$ is therefore satisfied.

We establish the assertion in question by an indirect argument (as in $[9$, Lemma 1.2.2.]). Suppose that, for any nonzero projection $P \leqq E$, there exists a nonzero projection $Q \leqq P$ such that $\rho(Q)>\omega_{z}(Q)$. We then define an ordered set of projections $\left\{Q_{\alpha}\right\}$ as follows: $Q_{1}$ is any nonzero projection $\leqq E$ such that $\rho\left(Q_{1}\right)>\omega_{z}\left(Q_{1}\right)$; suppose that $Q_{\alpha}$ is defined for $\alpha<\theta$ with the properties that $0<Q_{\alpha} \leqq E$, the $Q_{\alpha}$ are mutually orthogonal, and $\rho\left(Q_{\alpha}\right)>\omega_{z}\left(Q_{\alpha}\right)$; then if the projection $E_{\theta}=E-\sum_{\alpha<\theta} Q_{\alpha}$ is $0, Q_{\theta}$ is not defined, and otherwise, $Q_{\theta}$ is any nonzero projection $\leqq E_{\theta}$ such that $\rho\left(Q_{\theta}\right)>\omega_{z}\left(Q_{\theta}\right)$. The set $\left\{Q_{\alpha}\right\}$ so defined is countable, since $E$ is $\sigma$-finite, and writing $Q_{\alpha}=Q_{\alpha_{n}}$, we have $E=\sum_{n} Q_{\alpha_{n}}$ and $\rho(E)=\sum_{n} \rho\left(Q_{\alpha_{n}}\right)>\sum_{n} \omega_{z}\left(Q_{\alpha_{n}}\right)=\omega_{z}(E) \geqq \rho(E)$, a contradiction. The theorem is proved.

Roughly, the gist of this theorem is the following: if $\sigma$ and $\rho$ are CA states of $M$ in the relation $\sigma<\rho(M)$, then there exists a sequence $\left\{P_{n}\right\}$ of mutually orthogonal projections in $M$ such that $E_{\rho}=\sum_{n} P_{n}$ and $\sigma_{P_{n}} \ll \rho_{P_{n}}(M)$, for each $n$.

Remark 2.1. The trace $T$ on a finite factor $M$ is a CA state of $M$ satisfying the additional condition $T(A B)=T(B A)$, for all $A$ and $B$ in $M$. J. von Neumann has proved in [9] that $T$ has the form $\sum_{i=1}^{n} \omega_{x_{i}}$, for appropriate vectors $x_{i}$. We shall give here a simple proof of this result, based on Theorem 1. To this end, choose a nonzero projection $P$ in $M$ and a vector $x$ such that $T_{P}=\omega_{x}(M)$. We may assume that $P$ divides the identity operator $I$, in the sense that $I=\sum_{i=1}^{n} U_{i} P U_{i}^{*}$, where the $U_{i}$ are unitary operators in $M$ and the projections $U_{i} P U_{i}^{*}$ are mutually orthogonal. We compute $T(A)$ $=\sum_{i=1}^{n} T\left(A U_{i} P U_{i}^{*}\right)=\sum_{i=1}^{n} T\left(U_{i}^{*} A U_{i} P\right)=\sum_{i=1}^{n} T\left(P U_{i}^{*} A U_{i} P\right)=\sum_{i=1}^{n} \omega_{U_{i} x}(A)$, for each $A$ in $M$. This is the desired formula.

Strictly speaking, the notion of countable additivity is a lattice property of states. But, as we show now, it has topological implications.

Lemma 2.3. Let $\rho$ be a $C A$ state of the $\sigma$-finite $W^{*}$-algebra $M$, and let $\left\{A_{n}\right\}$ be a uniformly bounded sequence in $M$. Then $\rho\left(A_{n}^{*} A_{n}\right) \rightarrow 0$ if and only if $A_{n} E_{\rho} \rightarrow 0$ strongly. 
Proof. The proof will depend on the assertion $\left({ }^{*}\right)$ : there exist mutually orthogonal projections $P_{n}$ in $M$ and vectors $x_{n}$ and $y_{n}$ in $\mathfrak{S}$ such that $E$ $=\sum_{n} P_{n}, P_{n} y_{n}=x_{n}$, and the additional relations $\rho\left(A P_{n}\right)=\left(A x_{n}, y_{n}\right), \rho\left(A^{*} A\right)$ $\geqq\left\|A y_{n}\right\|^{2}$ hold for all $n$ and all $A$ in $M$.

To prove $\left({ }^{*}\right)$, we apply Theorem 1 exhaustively to obtain mutually orthogonal projections $P_{n}$ and vectors $x_{n}$, with $E_{\rho}=\sum_{n} P_{n}$ and $P_{n} x_{n}=x_{n}$, satisfying the relations $\rho_{P_{n}}=\omega_{x_{n}}(M)$. For each $n$, define a linear functional $f_{n}$ on the manifold $M x_{n}$ by the equation $f_{n}\left(A x_{n}\right)=\rho\left(A P_{n}\right)$; since $\left|\rho\left(A P_{n}\right)\right|^{2}$ $\leqq \rho(I)\left\|A x_{n}\right\|^{2}, f_{n}$ is well defined and bounded. By extending $f_{n}$ to $\left[M x_{n}\right]$ and applying the Riesz lemma, we can choose a vector $y_{n}$ in $\left[M x_{n}\right]$ such that $f_{n}\left(A x_{n}\right)=\left(A x_{n}, y_{n}\right)$. Next, choose a sequence $\left\{S_{n, i}\right\}$ in $M$ such that $\lim _{i} S_{n, i} x_{n}$ $=y_{n}$. Applying the Schwarz inequality, we obtain $\left|\left(A y_{n}, A y_{n}\right)\right|^{2}=$ $\lim _{i}\left|\rho\left(A^{*} A S_{n, i} P_{n}\right)\right|^{2} \leqq \rho\left(A^{*} A\right)\left(\lim _{i} \rho\left(P_{n} S_{n, i}^{*} A^{*} A S_{n, i} P_{n}\right)\right)=\rho\left(A^{*} A\right)\left(A y_{n}, A y_{n}\right)$. Therefore, $\left\|A y_{n}\right\|^{2} \geqq \rho\left(A^{*} A\right)$, and assertion $\left(^{*}\right)$ is proved.

Suppose now that $\rho\left(A_{i}^{*} A_{i}\right) \rightarrow 0$, for some uniformly bounded sequence $\left\{A_{i}\right\}$ in $M$. It follows by $\left({ }^{*}\right)$ that $A_{i} y_{n} \rightarrow 0$, for each $n$. Since the $A_{i}$ are uniformly bounded, it follows that $A_{i} z \rightarrow 0$, for each vector $z$ in the subspace $\left[M^{\prime} y_{n}\right]$. And by the same token, it follows that $A_{i} \rightarrow 0$ strongly on the subspace $\Sigma_{n} \oplus\left[M^{\prime} y_{n}\right]$. Let $Q$ be the projection on this subspace. For each $n$, we conclude from $\left(^{*}\right)$ that $\rho\left((I-Q) P_{n}\right)=0$. Therefore $(\rho(I-Q))^{2}$ $=\left(\rho\left((I-Q)\left(I-\sum_{n=1}^{N} P_{n}\right)\right)\right)^{2} \leqq \rho(I-Q) \rho\left(E_{\rho}-\sum_{n=1}^{N} P_{n}\right)$ for each $N$, and since $\lim _{N} \rho\left(E_{\rho}-\sum_{n=1}^{N} P_{n}\right)=0$, we conclude that $\rho(I-Q)=0$. This shows that $Q \geqq E_{\rho}$, and we have proved that $A_{i} E_{\rho} \rightarrow 0$ strongly.

Conversely, suppose that $A_{i} E_{\rho} \rightarrow 0$ strongly. If we choose the projections $P_{n}$ in $\left(^{*}\right)$ and write $Q_{N}=\sum_{n=1}^{N} P_{n}$, it follows explicitly that $\lim _{i} \rho\left(A_{i}^{*} A_{i} Q_{N}\right)$ $=0$, for each $N$. But since the $A_{i}$ are uniformly bounded, there exists a constant $K$ such that $\left|\rho\left(A_{i}^{*} A_{i}\right)-\rho\left(A_{i}^{*} A_{i} Q_{N}\right)\right|^{2} \leqq K \rho\left(I-Q_{N}\right)$, for all $i$ and $N$. It is trivial to conclude that $\lim _{i} \rho\left(A_{i}^{*} A_{i}\right)=0$. The lemma is proved.

Using the fact that a strongly convergent sequence is uniformly bounded, we obtain the following corollary to Lemma 2.3 : a state of a $\sigma$-finite $W^{*}$-algebra is CA if and only if it is strong sequentially continuous.

3. On cyclic projections in $W^{*}$-algebras. The crux of the duality theory for factors, as treated by Murray and von Neumann in [8, p. 174 et seq.], rests in the following (profound) question: given a $W^{*}$-algebra $M$ on $\mathfrak{S}$ and a vector $x$ in $\mathfrak{S}$, does each vector $y$ in the subspace $[M x]$ have the form $T x, T$ being a closed densely-defined operator belonging to $M$ ? A partial (and effective) answer is obtained by these authors [loc. cit. Lemma 9.2.1]: in any event, an arbitrary vector $y$ in $[M x]$ has the form $y=B T x$, where $B$ is a bounded operator in $M$ and $T$ is a closed densely-defined operator belonging to $M$. Descriptively, we shall call this result the "BT-Theorem," and the first result, when valid, the "T-Theorem" for $[M x]$.

As it develops, this very question is the crux of the Radon-Nikodým theorem in the cyclic case, $\rho<\omega_{x}(M)$. In fact, the theorem " $\omega_{y}<\omega_{x}(M)$ im- 
plies $\omega_{y}=\omega_{T x}(M)$, for all such $y$ " already implies the T-Theorem for [Mx]. By means of the BT-Theorem and a trace argument, one can easily show that the finiteness of the projection $E_{[M x]}$ in $M^{\prime}$ is sufficient for the validity of the T-Theorem for $[M x]$. In this section, we prove (in Theorem 2) that the finiteness of $E_{[M x]}$ is also necessary for the strict validity of the T-Theorem on $[M x]$, or even on $\left[M^{\prime} x\right]$; and in turn, that this is necessary and sufficient for the validity of the Radon-Nikodým theorem in the cyclic case, for either $M$ or $M^{\prime}$. Our proof, incidentally, makes no use of the trace, but does depend in part on techniques of Murray and von Neumann.

Notation 3.1. Let $M$ be a $W^{*}$-algebra on $\mathfrak{S}$. We recall first some concepts from the dimension theory for $M$ (see [6] and [8]). By a partial isometry $V$ in $M$ we mean an operator in $M$ such that both $V^{*} V$ and $V V^{*}$ belong to $M_{P}$; the range of $V^{*} V$ is called the initial domain of $V$, and the range of $V V^{*}$ is called the terminal domain of $V$. Two projections $P$ and $Q$ in $M$ are called equivalent $\bmod M$ (symbolically, $P \sim Q(M)$ ) if there exists a partial isometry $V$ in $M$ such that $V^{*} V=P$ and $V V^{*}=Q$. If only $V V^{*} \leqq Q$, we say then that $P$ is equivalent to part of $Q \bmod M$, and write $P<Q(M)$. It is known that $P<Q(M)$ and $Q<P(M)$ together imply $P \sim Q(M)$. A projection $P$ in $M$ is called finite if $P$ is not equivalent $\bmod M$ to any projection $Q<P$, infinite if $P$ is not finite, and purely infinite if there is no finite projection $Q$ in $M$ less than $P$. The $W^{*}$-algebra $M$ itself is finite, infinite, or purely infinite according as the identity $I$ in $M$ is finite, infinite, or purely infinite.

A closed densely-defined operator $T$ on $\mathfrak{S}$ is said to "belong to $M$ " (symbolically, $T \eta M$ ) if $T U=U T$, for each unitary operator $U$ in $M^{\prime}$. (The following criterion is often useful: if $M_{0}^{\prime}$ is any strongly dense subalgebra of $M^{\prime}$, then a closed densely-defined operator $T$ belongs to $M$ if and only if $T A \supseteq A T$, for each $A$ in $M_{0}^{\prime}$.) Throughout our work, we shall denote by $C(M)$ the collection of all closed densely-defined operators on $\mathfrak{S}$ belonging to $M$. An important technique in treating elements of $C(M)$ is the polar decomposition: each $T$ in $C(M)$ can be written in one and only one way as a product $V H$, where $H$ is a (possibly unbounded) non-negative SA operator in $C(M)$, and where $V$ is a partial isometry in $M$ with initial domain $=[$ Range $H]$ and terminal domain $=[$ Range $T]$.

We shall now formally describe the concept involved in the T-Theorem.

Definition 3.1. Let $M$ be a $W^{*}$-algebra on $\mathfrak{S}$ and $z$ a vector in $\mathfrak{S}$. A vector $x$ is integrable $(M, z)$ if there exists a $T$ in $C(M)$ such that $x=T z$. (Necessarily, of course, $x \in[M z]$.) More generally, a state $\rho$ of $M$ is called integrable $\left(M^{\prime}, z\right)$ if there exists a vector $x$ integrable $\left(M^{\prime}, z\right)$ such that $\rho$ $=\omega_{x}(M)$.

For ad hoc reasons, the term "square-integrable" might be preferred. Consider, for example, a maximal abelian $W^{*}$-algebra $M$ and a normalized cyclic vector $z$ for $M$; by one form of the spectral theorem, we know that $M$ is isomorphic to the space of all continuous functions $C(X)$ on some compact 
hausdorff space $X$. Viewed as a state of $C(X), \omega_{z}$ induces a regular probability measure $\mu$ on $X$, and it is easily seen that $[M z]$ is unitarily equivalent to $L_{2}(X, \mu)$.

A vague point in Definition 3.1 is cleared up by the following lemma, which shows that $x$ integrable $\left(M^{\prime}, z\right)$ and $\omega_{y}=\omega_{x}(M)$ imply that $y$ is also integrable $\left(M^{\prime}, z\right)$.

Lemma 3.1. The vector $x$ is integrable $\left(M^{\prime}, z\right)$ if and only if there exists a $S A$ operator $H$ in $C\left(M^{\prime}\right)$, with $[$ Range $H] \subseteq[M z]$, such that $\omega_{x}=\omega_{H z}(M)$.

Proof. Assume first that $x$ is integrable $\left(M^{\prime}, z\right)$, so that $x=T z$, for some $T$ in $C\left(M^{\prime}\right)$. Since both $T E_{[M z]}$ and its adjoint $\left(T E_{[M z]}\right)^{*} \supseteq E_{[M z]} T^{*}$ are densely-defined, the operator $T E_{[M z]}$ has a closed extension $\bar{T}$, which obviously belongs to $M^{\prime}$ and satisfies $x=\bar{T} z$. Applying the polar decomposition, write $\bar{T}=V H, H$ being a SA operator in $C\left(M^{\prime}\right)$ and $V$ a partial isometry in $M^{\prime}$ with initial domain $=[$ Range $H]$. We have $V H=V H E_{[M z]}$, so $V^{*} V H E_{[M z]}=H E_{[M z]}=H$, and hence $\left(H E_{[M z]}\right)^{*}=E_{[M z]} H=H$; in other words, [Range $H] \subseteq[M z]$, and obviously, $\omega_{x}=\omega_{H z}(M)$.

Conversely, if $\omega_{x}=\omega_{H_{z}}(M)$, we must show that $x=T z$, for some $T$ in $C(M)$. Now, the fact that $\|A x\|^{2}=\|A H z\|^{2}$, for all $A$ in $M$, implies that the operator $V_{0}$ defined on the manifold $M(H z)$ by the equation $V_{0}(A H z)=A x$ $\left(A\right.$ in $M$ ) is isometric. Obviously, $V_{0}$ extends to a partial isometry $V$ in $M^{\prime}$ with initial domain $[M(H z)]$ and terminal domain $[M x]$. Let $T=V H$. It is easy to verify that $T \in C\left(M^{\prime}\right)$, and by definition, $x=T z$.

The next two lemmas are patterned after the Murray-von Neumann proof of the BT-Theorem (and involve ideas due originally to Friedrichs). Though we could obviate these lemmas by alterations later on, they have some independent interest. In particular, Lemma 3.2 already yields the Radon-Nikodým theorem in the cyclic case for a maximal abelian ring, it being easy to convert the prescribed convergence condition into the usual absolute continuity relation.

Lemma 3.2. A state $\rho$ of the $W^{*}$-algebra $M$ is integrable $\left(M^{\prime}, z\right)$ if (and only if), for each sequence $\left\{A_{n}\right\}$ in $M$ with the properties that $\lim _{n} A_{n} z=0$ and $\lim _{n, m} \rho\left(\left(A_{n}-A_{m}\right)^{*}\left(A_{n}-A_{m}\right)\right)=0$, it results also that $\lim _{n} \rho\left(A_{n}^{*} A_{n}\right)=0$.

Proof. Define a bilinear form $Q(A z, B z)$ on the manifold $M z$ in $\mathfrak{S}$ by setting $Q(A z, B z)=(A z, B z)+\rho\left(B^{*} A\right)$. The properties of the state $\rho$ and the original inner product $($,$) on \mathfrak{S}$ together imply that $Q($,$) is an inner product. Let$ $\mathfrak{M}$ denote the completion of $M z$ in this new inner product. We see, by our definition, that there is a natural mapping $\phi$ from $\mathfrak{M}$ in $\mathfrak{S}$, defined as follows: if a vector $\xi$ in $\mathfrak{M}$ is a limit (in the new inner product) of a sequence $\left\{A_{n} z\right\}$, then $\phi(\xi)=\lim _{n} A_{n} z$ (in the original inner product). In this context, as the reader can easily check, the convergence condition on $\rho$ is equivalent to the following condition: the natural mapping $\phi$ is one-one. In what follows, therefore, 
we can identify $\mathfrak{M}$ with $\phi(\mathfrak{M})(\subseteq[M z])$. The new norm $(Q(x, x))^{1 / 2}$ of an element $x$ in $\mathfrak{M}$ will be denoted $\|x\|_{0}$, and the (original) norm of any $x$ in $\mathfrak{S}$ will be denoted $\|x\|$.

By virtue of the inequality $|(y, x)| \leqq\|y\|\left\|_{0}\right\| x \|$, for any $x$ in $[M z]$ and $y$ in $\mathfrak{M}$, we see that $f(y)=(y, x)$ is a bounded linear functional on $\mathfrak{M}$. According to the Riesz lemma, there exists a unique $x^{*}$ in $\mathfrak{M}$ such that $(y, x)=Q\left(y, x^{*}\right)$, for all $y$ in $\mathfrak{M}$. We assert that the operator $H$ defined on $[M z]$ by the equation $H x=x^{*}$ is bounded and positive SA; in fact,

$$
(H x, y)=Q\left(x^{*}, y^{*}\right)=\bar{Q}\left(y^{*}, x^{*}\right)=(x, H y),
$$

so $H$ is symmetric, and $(H x, x)=Q\left(x^{*}, x^{*}\right)=0, x^{*}$ being 0 only if $(y, x)=0$, for all $y$ in $\mathfrak{M}$, or consequently, only if $x=0$, so $H$ is positive; and the assertion follows from the fact that an everywhere defined symmetric operator is bounded SA.

We prove next that $H(A x)=A(H x)$, for all $A$ in $M$ and $x$ in [Mz], or what is the same, that $(A x)^{*}=A x^{*}$. Observe that $\mathfrak{M}$ reduces $M$; in fact, if $\left\{A_{n}\right\}$ is $\rho$-cauchy (in the sense that $\left.\lim _{n, m} \rho\left(\left(A_{n}-A_{m}\right) *\left(A_{n}-A_{m}\right)\right)=0\right)$, then so is $\left\{A A_{n}\right\}$, for each $A$ in $M$-as follows from the inequalities $K \rho\left(\left(A_{n}-A_{m}\right) *\left(A_{n}-A_{m}\right)\right) \geqq \rho\left(\left(A_{n}-A_{m}\right) * A^{*} A\left(A_{n}-A_{m}\right)\right), K$ being a constant so chosen that $K I \geqq A^{*} A$-therefore, if $\left\{A_{n} z\right\}$ is $\|_{0}$-cauchy, so is $\left\{A A_{n} z\right\} ;$ likewise if $\left\{A_{n} z\right\}$ and $\left\{B_{n} z\right\}$ are $\|_{0}$-equivalent, then so are $\left\{A A_{n} z\right\}$ and $\left\{A B_{n} z\right\}$; and these two facts obviously secure our assertion. By similar reasoning, we see that the contractions of $A$ and $A^{*}$ to $\mathfrak{M}$ are $\|_{0}$-bounded. Now, by definition of $Q(),, Q(A B z, C z)=Q\left(B z, A^{*} C z\right)$, for all $A, B, C$ in $M$. Therefore, by continuity, $Q(A x, y)=Q\left(x, A^{*} y\right)$, for all $x$ and $y$ in $\mathfrak{M}$. Using this fact, we have $Q\left(y,(A x)^{*}\right)=(y, A x)=\left(A^{*} y, x\right)=Q\left(A^{*} y, x^{*}\right)$ $=Q\left(y, A x^{*}\right)$. Therefore, $(A x)^{*}=A x^{*}$, as asserted.

Being a positive SA operation on $[M z], H$ will have a positive SA inverse $H^{-1}$ on $[M z]$. Notice that $H^{-1} \geqq I$ (really, $E_{[M z]}$ ) on Domain $H^{-1}=$ Range $H$, because $\left(H^{-1} x^{*}, x^{*}\right)=\left(x, x^{*}\right)=Q\left(x^{*}, x^{*}\right) \geqq\left(x^{*}, x^{*}\right)$. Therefore, by the spectral theorem, $\left(H^{-1}-I\right)$ has a positive SA square root $T$ on $[M z]$, and Domain $T^{2}=$ Domain $\left(H^{-1}-I\right)=$ Domain $H^{-1}$. Also, since $T$ is a baire function of $H$, and since $H$ commutes with all elements of $M$ on $[M z]$, it follows likewise that $A T x=T A x$, for all $x$ in Domain $T$ and all $A$ in $M$.

Choose a sequence $\left\{x_{n}\right\}$ in Range $H$ which converges to $z$ in the $\|_{0}$-norm (noting that the range of $H$ is $\|_{0}$-dense in $\mathfrak{M}$ ). These vectors $x_{n} \in$ Domain $T^{2}$ $\subseteq$ Domain $T$, and we have $\left(T\left(x_{n}-x_{m}\right), T\left(x_{n}-x_{m}\right)\right)=\left(x_{n}-x_{m}, T^{2}\left(x_{n}-x_{m}\right)\right)$ $=\left(x_{n}-x_{m}, H^{-1}\left(x_{n}-x_{m}\right)\right)-\left\|x_{n}-x_{m}\right\|^{2}=\left\|x_{n}-x_{m}\right\|_{0}^{2}-\left\|x_{n}-x_{m}\right\|^{2} \rightarrow 0$. Since $T$ is a closed operator, this implies $z \in$ Domain $T$ and $\lim _{n} T x_{n}=z$. Therefore, on the one hand, $\lim _{n}\left(A T x_{n}, T x_{n}\right)=(A T z, T z)$, and on the other hand, $\lim _{n}\left(A T x_{n}, T x_{n}\right)=\lim _{n}\left(T A x_{n}, T x_{n}\right)=\lim _{n}\left(\left(A x_{n}, H^{-1} x_{n}\right)-\left(A x_{n}, x_{n}\right)\right)$ $=\lim _{n}\left(Q\left(A x_{n}, x_{n}\right)-\left(A x_{n}, x_{n}\right)\right)=Q(A z, z)-(A z, z)=\rho(A)$. It results that $\rho=\omega_{T z}(M)$. Finally, by setting $T=0$ on the subspace $\mathfrak{S} \theta[M z]$, we obtain 
$T \in C\left(M^{\prime}\right)$, and the proof is completed.

LеммA 3.3. Let $\left\{\rho_{i}\right\}$ be a sequence of states of the $W^{*}$-algebra $M$ which are integrable $\left(M^{\prime}, z\right)$ and which satisfy $\sum_{i=1}^{\infty} \rho_{i}(I)<\infty$. Then the state $\rho$ of $M$ defined by the equation $\rho(A)=\sum_{i=1}^{\infty} \rho_{i}(A)$ is itself integrable $\left(M^{\prime}, z\right)$.

Proof. It will suffice to prove that $\rho$ satisfies the convergence condition of Lemma 3.2 -that is, assuming that $\lim _{n} A_{n} z=0$ and $\lim _{n, m} \rho\left(\left(A_{n}-A_{m}\right)^{*}\left(A_{n}-A_{m}\right)\right)=0$, for some fixed sequence $\left\{A_{n}\right\}$ in $M$, to show that $\lim _{n} \rho\left(A_{n}^{*} A_{n}\right)=0$. Consider such a sequence. As follows easily from the Schwarz inequality, $\rho\left(A_{n}^{*} A_{n}\right) \leqq \rho\left(A_{m}^{*} A_{m}\right)+\rho\left(\left(A_{n}-A_{m}\right) *\left(A_{n}-A_{m}\right)\right)$ $+2\left[\rho\left(A_{m}^{*} A_{m}\right) \rho\left(\left(A_{n}-A_{m}\right)^{*}\left(A_{n}-A_{m}\right)\right)\right]^{1 / 2}$, for all $m$ and $n$. This shows that the numbers $\rho\left(A_{n}^{*} A_{n}\right)$ are uniformly bounded, and moreover, that if some subsequence of the $\rho\left(A_{n}^{*} A_{n}\right)$ tends to 0 , then so does the original sequence. We shall exhibit such a subsequence.

By definition, we may write $\rho_{i}=\omega_{y_{i}}(M)$ for each $i$, where $y_{i}$ is a vector integrable $\left(M^{\prime}, z\right)$. Now, extract a subsequence $\left\{B_{n}\right\}$ of the $\left\{A_{n}\right\}$ such that $\left\|B_{n} y_{i}\right\|^{2} \leqq 1 / 2^{i}$, whenever $i \leqq n$. We shall prove, by an indirect argument, that $\lim _{n} \rho\left(B_{n}^{*} B_{n}\right)=0$. Suppose that there exists a $\delta>0$ with the following property: given $N, \delta<\sum_{i=N}^{\infty}\left\|B_{n} y_{i}\right\|^{2}$, for all sufficiently large $n$. Then, for each $n$ sufficiently large, there exists a least integer $M_{n}$ such that $\delta / 2$ $\leqq \sum_{i=N}^{M_{n}}\left\|B_{n} y_{i}\right\|^{2}$. We assert that $n$ can be chosen so that $M_{n} \leqq n$; in fact, otherwise $\sum_{i=N}^{n-1}\left\|B_{n} y_{i}\right\|^{2}<\delta / 2$, for all $n$ sufficiently large, so $\sum_{i=n}^{\infty}\left\|B_{n} y_{i}\right\|^{2}$ $>\delta / 2$; but this is impossible, since $\lim _{n} \sum_{i=n}^{\infty}\left\|B_{n} y_{i}\right\|^{2}=0$ (as one sees by choosing $m$ and $k$ properly in the inequality $\sum_{i=n}^{\infty}\left\|B_{n} y_{i}\right\|^{2}$ $=2\left[\sum_{i=1}^{\infty}\left\|\left(B_{n}-B_{m}\right) y_{i}\right\|^{2}+\sum_{i=\mathbf{k}}^{\infty}\left\|B_{m} y_{i}\right\|^{2}\right]$, valid for all $\left.k \leqq n\right)$. This being so, one can therefore construct a divergent series $\sum_{i=1}^{\infty}\left\|B_{\phi(i)} y_{i}\right\|^{2}$, in which the index $\phi(i)$ is not less than $i$, for each $i$. This is a contradiction, however, by virtue of our choice of the subsequence $\left\{B_{n}\right\}$. Therefore, no such $\delta$ exists, and given any $\epsilon>0$, we can find an $N$ such that $\sum_{i=N}^{\infty}\left\|B_{n} y_{i}\right\|^{2}<\epsilon / 2$, for some $n$ arbitrarily large. Moreover, $\sum_{i=1}^{N}\left\|B_{n} y_{i}\right\|^{2}<\epsilon / 2$, for $n$ sufficiently large, as follows from our assumption that each $y_{i}$ is integrable $\left(M^{\prime}, z\right)$. Therefore, for such $n, \rho\left(B_{n}^{*} B_{n}\right)=\sum_{i=1}^{\infty}\left\|B_{n} y_{i}\right\|^{2}<\epsilon$, completing the proof.

Both the T-Theorem for $[M z]$ and the Radon-Nikodým theorem in the cyclic case $\rho<\omega_{z}(M)$ hinge on the existence of certain duality relationships between $[M z]$ and $\left[M^{\prime} z\right]$, as described in the following (fundamental) lemma; in Theorem 2 , we correlate these notions with the dimension theory. In stating this lemma, by the $d u a l$ of a condition on a $W^{*}$-algebra $M$ we shall mean the same condition stated for the commutant $M^{\prime}$ of $M$.

Lemma 3.4. The following conditions on a $W^{*}$-algebra $M$ and a vector $z$ in Sc are equivalent:

(1) If $Q^{\prime}$ is a projection in $M^{\prime}$ satisfying $0 \leqq Q^{\prime}<E_{[M z]}$, then $\left[M^{\prime} Q^{\prime} z\right]$ $\subset\left[M^{\prime} z\right]$ properly; 
(2) If $Q^{\prime}$ is a projection in $M^{\prime}$ satisfying $0 \leqq Q^{\prime} \leqq E_{[M z]}$, then there exists a projection $Q$ in $M$ such that $[M Q z]=\left[M Q^{\prime} z\right]$;

(3) If $x$ is integrable $\left(M^{\prime}, z\right)$ and if $\omega_{y} \ll \omega_{x}(M)$, then $y$ is integrable $\left(M^{\prime}, z\right)$;

(4) Each vector in $\left[M^{\prime} z\right]$ is integrable $\left(M^{\prime}, z\right)$;

(5) If $\rho$ is any $C A$ state of $M$ satisfying $\rho<\omega_{z}(M)$, then there exists $a$ vector $x$ integrable $\left(M^{\prime}, z\right)$, such that $\rho=\omega_{x}(M)$;

and each of the dual conditions $\left(1^{\prime}\right),\left(2^{\prime}\right),\left(3^{\prime}\right),\left(4^{\prime}\right)$, and $\left(5^{\prime}\right)$.

Proof. The scheme of proof runs as follows: we show first that (1) implies (2); thereupon, we demonstrate the equivalence of (2), (3), (4) and their duals; next, these are proved equivalent to (1) and $\left(1^{\prime}\right)$; and finally, the equivalence of these conditions with $(5)$ and $\left(5^{\prime}\right)$ is proved.

$(1) \rightarrow(2)$ : Observe first that (1) implies the following condition $\left(^{*}\right)$ : for any nonzero projection $Q^{\prime}$ in $M^{\prime}$ satisfying $Q^{\prime} \leqq E_{[M z]}$, there exists a projection $R$ in $M$ such that $R z \neq 0$ and $Q^{\prime} R z=R z$. In fact, the projection $R^{\prime}=E_{[M z]}$ $-Q^{\prime}$ is (strictly) less than $E_{[M z]}$, and so by (1), the projection $R$ on $\left[M^{\prime} z\right]$ $\vartheta\left[M^{\prime} R^{\prime} z\right]$ is nonzero. Hence $R z \neq 0$ and $R z-Q^{\prime} R z=R R^{\prime} z=0$, as desired. We prove in turn that $\left(^{*}\right)$ implies (2).

If $Q^{\prime}=0$, we put $Q=0$. Otherwise we define $Q$ by the following transfinite induction. Let $Q_{1}$ be any projection in $M$ such that $Q_{1} z \neq 0$ and $Q^{\prime} Q_{1} z=Q_{1} z$ (by $\left.\left({ }^{*}\right)\right)$. So then, $\left[M Q_{1} z\right] \subseteq\left[M Q^{\prime} z\right]$. Suppose that $Q_{\alpha}$ is defined for all $\alpha<\theta$ with the properties that the subspaces $\left[M Q_{\alpha} z\right]$ are non-null, mutually orthogonal, and contained in $\left[M Q^{\prime} z\right]$. Let $R_{\theta}^{\prime}$ be the projection on $\sum_{\alpha<\theta}$ $\oplus\left[M Q_{\alpha} z\right]$. If $Q^{\prime}-R_{\theta}^{\prime}=0$, then $Q_{\theta}$ is not defined. Otherwise, apply $\left(^{*}\right)$ to the projection $Q^{\prime}-R_{\theta}^{\prime}$ in place of $Q^{\prime}$ to obtain a projection $Q_{\theta}$ in $M$ such that $Q_{\theta} z \neq 0$ and $\left(Q^{\prime}-R_{\theta}^{\prime}\right) Q_{\theta} z=Q_{\theta} z$. It results that $\left[M Q_{\theta} z\right] \subseteq\left[M\left(Q^{\prime}-R_{\theta}^{\prime}\right) z\right]=\left[M Q^{\prime} z\right]$ $\ominus \sum_{\alpha<\theta} \oplus\left[M Q_{\alpha} z\right]$, so therefore $\left[M Q_{\theta} z\right]$ is orthogonal to the subspaces $\left[M Q_{\alpha} z\right]$, for $\alpha<\theta$. Since the projection $\left[M Q^{\prime} z\right]$ is $\sigma$-finite, we can enumerate the $Q_{\alpha}$ so defined, and it follows that $\left[M Q^{\prime} z\right]=\sum_{n} \oplus\left[M Q_{\alpha_{n}} z\right]$. Let $Q$ $=\mathrm{V}_{n} Q_{\alpha_{n}}$. Since $\left[M Q_{\alpha_{n}} z\right] \subseteq[M Q z]$ for all $n$, we have $\left[M Q^{\prime} z\right] \subseteq[M Q z]$. On the other hand, $Q^{\prime} Q_{\alpha_{n}} z=Q_{\alpha_{n}} z$, so that $\left(Q_{\alpha_{n}}\right)\left(I-Q^{\prime}\right) z=0$, for all $n$. Hence $\left(Q_{\alpha_{1}} \bigvee \cdots \vee Q_{\alpha_{n}}\right)\left(I-Q^{\prime}\right) z=0$, for all $n$. Hence $\left(I-Q^{\prime}\right) Q z=Q\left(I-Q^{\prime}\right) z=0$, so $\left(I-Q^{\prime}\right)[M Q z]=0$. Therefore, $[M Q z] \subseteq\left[M Q^{\prime} z\right]$, so that equality follows, proving (2).

$(2) \rightarrow(3):$ By Lemma 3.1, we can assume that $x=H z$, where $H$ is a SA operator belonging to $M^{\prime}$ with $[$ Range $H] \subseteq[M z]$. By Lemma 2.2, $\omega_{y}=\omega_{A x}(M)$, for some bounded operator $A$ in $M^{\prime}$. Again by Lemma 3.1, we can assume that $A$ is bounded SA with [Range $A] \subseteq[M x](\subseteq[M z])$. Condition (3) will clearly follow if we can prove that the linear operator $A H$ has a closed extension.

We shall prove first that $P^{\prime} H$ has a closed extension, where $P^{\prime}$ is any projection in $M^{\prime}$ such that $P^{\prime} \leqq E_{[M z]}$. To do this, it will suffice to prove that $\left(P^{\prime} H\right)^{*}=H P^{\prime}$ is densely defined. In turn, since $P^{\prime} \mathfrak{S}=P^{\prime}[M z]=\left[M P^{\prime} z\right]$, it will suffice to prove that the manifold (Domain $H) \cap\left[M P^{\prime} z\right]$ is dense in 
[MP'z]. Using condition (2), choose a projection $P$ in $M$ such that $[M P z]$ $=\left[M P^{\prime} z\right]$. Since $z \in$ Domain $H$, and since $H \in C\left(M^{\prime}\right)$, the manifold $M P z$ is contained in Domain $H$. Therefore, (Domain $H) \cap\left[M P^{\prime} z\right] \supseteq M P z$, which is dense in $\left[M P^{\prime} z\right]$. It follows that $P^{\prime} H$ has a closed extension (in $C\left(M^{\prime}\right)$, of course).

Turning to the general case, we shall show that $A H$ satisfies the following obvious criterion for the existence of a closed extension: if $\left\{x_{n}\right\}$ is any sequence of vectors in Domain $A H$ such that $x_{n} \rightarrow 0$ and $A H x_{n} \rightarrow y$, then necessarily $y=0$. To this end, let $\mathfrak{M}=[v$ in $[M x] \mid A v=0]$, and let $P=E_{[M x]}-E_{\mathfrak{M}}$. It is easy to see that $P \in\left(M^{\prime}\right)_{P}$ and that $P A=A P$. Furthermore, let $E(\lambda)$ be the spectral resolution of $A$ and let $\delta_{n}$ denote the interval $(-\infty,-1 / n)$ $\cup(1 / n,+\infty)$. Set $A_{n}=\int_{\delta_{n}} \lambda d\left(P E_{\lambda}\right)$ and $E_{n}=\int_{\delta_{n}} d\left(P E_{\lambda}\right)$. It follows easily from our definitions that $A_{n}$ has a bounded inverse on $E_{n} \mathfrak{S}$ and that $\mathrm{V}_{n} E_{n}$ $=E_{[\text {Range } A]}(\subseteq[M z])$. Now then, if $\lim _{i} A H x_{i}=y$, then $\lim _{i} A_{n}\left(E_{n} H\right) x_{i}$ $=E_{n} y$, so that $\lim _{i}\left(E_{n} H\right) x_{i}=A_{n}^{-1} E_{n} y$. Since $E_{n} H$ has a closed extension, by the preceding paragraph, it is easy to conclude that $A_{n}^{-1} E_{n} y=0$. Therefore, $E_{n} y=0$, for all $n$, so that $y=E_{\text {[Range } A]} y=0$. Condition 3 is therefore established.

$(3) \rightarrow(4)$ : The BT-Theorem as cited above can be stated as follows: given $y$ in $\left[M^{\prime} z\right]$, there exists an $x$ integrable $\left(M^{\prime}, z\right)$ such that $\omega_{y} \ll \omega_{x}(M)$. According to (3), this means that $y$ is itself integrable $\left(M^{\prime}, z\right)$.

$(4) \rightarrow\left(2^{\prime}\right)$ : In fact, let $Q$ be a projection in $M$ satisfying $0 \leqq Q \leqq E_{\left[M^{\prime} z\right]}$. By dualizing the proof of the implication (1) $\rightarrow(2)$, we see it will suffice to exhibit a projection $R^{\prime}$ in $M^{\prime}$ such that $R^{\prime} z \neq 0$ and $R^{\prime} z \in\left[M^{\prime} Q z\right]$. Now, the vector $Q z \in\left[M^{\prime} z\right]$, and it is therefore integrable $\left(M^{\prime}, z\right)$ by assumption. We write $Q z=T z$, for some $T$ in $C\left(M^{\prime}\right)$, and observe that $A T z \in\left[M^{\prime} Q z\right]$, for each $A$ in $M^{\prime}$. If $T=V H$ is the polar decomposition of $T$, then also $A H z$ $\in\left[M^{\prime} Q_{z}\right]$, for each $A$ in $M^{\prime}$. But, by the spectral theorem, we can obviously choose an $A$ in $M^{\prime}$ with the property that $A H z=R^{\prime} z \neq 0, R^{\prime}$ being some projection in $M^{\prime}$, and for such an $A$, we also have $\left(A V^{*}\right) T z=R^{\prime} z$. The implication is verified.

By duality, it now follows that $\left(2^{\prime}\right) \rightarrow\left(3^{\prime}\right) \rightarrow\left(4^{\prime}\right) \rightarrow(2)$. We shall complete the first part of the proof by showing that conditions (2), (3), (4) and their duals imply (1) and $\left(1^{\prime}\right)$.

Suppose that (1) fails. Then there exists in $M^{\prime}$ a projection $Q_{0}^{\prime}, Q_{0}^{\prime}<E_{[M z]}$, such that $\left[M^{\prime} Q_{0}^{\prime} z\right]=\left[M^{\prime} z\right]$. Now if condition (2) holds for the vector $z$, it obviously holds for the vector $Q_{0}^{\prime} z$ and projections $Q^{\prime} \leqq Q_{0}^{\prime}$. Moreover, each vector in $\left[M^{\prime} Q_{0}^{\prime} z\right]$ will then be integrable $\left(M^{\prime}, Q_{0}^{\prime} z\right)$. Since we are assuming that $z \in\left[M^{\prime} Q_{0}^{\prime} z\right]$, we have $z=T\left(Q_{0}^{\prime} z\right)$, for some $T$ in $C\left(M^{\prime}\right)$. Furthermore, $\left(I-Q_{0}^{\prime}\right) z$ is a nonzero vector in $[M z]$, and we can choose a sequence $\left\{A_{n}\right\}$ in $M$ such that $\lim _{n} A_{n} z=\left(I-Q_{0}^{\prime}\right) z$. We have $\lim _{n} A_{n} Q_{0}^{\prime} z=0$ and $\lim _{n} T\left(A_{n} Q_{0}^{\prime} z\right)$ $=\left(I-Q_{0}^{\prime}\right) z$. Since $T$ is assumed to be closed, we must also have $\left(I-Q_{0}^{\prime}\right) z=0$, a contradiction. Therefore, $(2) \rightarrow(1)$, and dually, $\left(2^{\prime}\right) \rightarrow\left(1^{\prime}\right)\left(\rightarrow\left(2^{\prime}\right)\right)$. 
It is obvious that $(5) \rightarrow(4)$. We shall complete the proof by showing that $(3) \rightarrow(5)$. We shall assume for convenience that the CA state $\rho$ is normalized.

First, we prove an assertion $\left({ }^{* *}\right)$ : given $n$ mutually orthogonal projections $P_{1}, \cdots, P_{n}$ in $M$ with the property that each state $\rho_{P_{i}}$ is integrable $\left(M^{\prime}, z\right)$, then the state $\rho_{\left(P_{1}+\cdots+P_{n}\right)}$ of $M$ is itself integrable $\left(M^{\prime}, z\right)$. In fact, let $\tau$ $=\sum_{i=1}^{n} \rho_{P_{i}}(M)$, and write $R=P_{1}+\cdots+P_{n}$. By Lemma 3.3, $\tau$ is integrable $\left(M^{\prime}, z\right)$. The assertion $\left({ }^{* *}\right)$ will follow from (3) if we show that $\rho_{R} \ll \tau(M)$. In turn, this will follow if we show that $\rho_{R}(A) \leqq\left[\sum_{i=1}^{n}\left(\rho_{P_{i}}(A)\right)^{1 / 2}\right]^{2}$, for each non-negative SA $A$ in $M$. But, for such an $A, \rho_{R}(A)=\sum_{i=1}^{n} \rho_{R}\left(A P_{i}\right)$ $\leqq \sum_{i=1}^{n}\left|\rho_{R}\left(\left(A^{1 / 2}\right)\left(A^{1 / 2} P_{i}\right)\right)\right| \leqq\left[\rho_{R}(A)\right]^{1 / 2}\left[\sum_{i=1}^{n}\left(\rho\left(P_{i} A P_{i}\right)\right)^{1 / 2}\right]$, by the Schwarz inequality, and the desired inequality follows.

Next, we show there exists an increasing sequence $\left\{Q_{n}\right\}$ in $M_{P}$ such that $E_{\rho}=\sum_{n} Q_{n}, \rho\left(I-Q_{n}\right) \leqq 1 / 4^{n}$, and $\rho_{\left(Q_{n+1}-Q_{n}\right)}$ is integrable $\left(M^{\prime}, z\right)$, for each $n$. To see this, apply Theorem 1 exhaustively to obtain a sequence $\left\{P_{n}\right\}$ in $M_{P}$ such that $E_{\rho}=\sum_{n} P_{n}$ and $\rho_{P_{n}}=\omega_{x_{n}}(M)$, for some vector $x_{n}$ in $\left[M^{\prime} z\right]$. By (4), which is implied by (3), the $\rho_{P_{n}}$ are integrable $\left(M^{\prime}, z\right)$. Now, choose an index $n_{1}$ such that $\rho\left(I-\left(P_{1}+\cdots+P_{n_{1}}\right)\right) \leqq 1 / 4$, and set $Q_{1}=P_{1}+\cdots$ $+P_{n_{1}}$. Then, choose an index $n_{2}$ exceeding $n_{1}$ such that $\rho\left(I-\left(P_{1}+\cdots+P_{n_{2}}\right)\right)$ $\leqq 1 / 4^{2}$, and set $Q_{2}=P_{1}+\cdots+P_{n_{2}}$. Proceed in this fashion to define the $Q_{n}$. Now, $\rho_{\left(Q_{m+1}-Q_{m}\right)}=\rho_{\left(P_{n_{m}+1}+\cdots+P_{n_{m}+1}\right)}$, and by the assertion $(* *)$, this state is integrable $\left(M^{\prime}, z\right)$.

Finally, let $R_{n}=Q_{n}-Q_{n-1}$ when $n \geqq 2$, and $R_{1}=Q_{1}$. We have $\rho\left(R_{n}\right)$ $\leqq \rho\left(I-Q_{n}\right)+\rho\left(I-Q_{n-1}\right) \leqq 5 / 4^{n}$, so that $\sum_{n} 2^{n} \rho_{R_{n}}(I)<\infty$. But the states $2^{n} \rho_{R_{n}}$ are integrable $\left(M^{\prime}, z\right)$ by construction, and we may therefore apply Lemma 3.3 to conclude that the state $\lambda=\sum_{n}{ }^{n}{ }^{n} \rho_{R_{n}}(M)$ is itself integrable $\left(M^{\prime}, z\right)$. We assert that $\rho \ll \lambda(M)$. In fact, consider any non-negative SA $A$ in $M$ such that $\lambda(A) \leqq 1$. It follows that $\left[\rho_{R_{n}}(A)\right]^{1 / 2} \leqq \lambda(A) /\left(2^{1 / 2}\right)^{n}$. Putting $K=\sum_{n=1}^{\infty}\left[1 /\left(2^{1 / 2}\right)\right]^{n}$, we conclude from the inequality in $\left({ }^{* *}\right)$ that $\rho_{Q_{n}}(A)$ $\leqq K^{2} \lambda(A)$, for all $n$. Therefore, since $\rho$ is strong sequentially continuous, $\rho(A) \leqq K^{2} \lambda(A)$. In other words, $\rho \ll \lambda(M)$. By condition (3), $\rho$ is integrable $\left(M^{\prime}, z\right)$, as desired. This completes the proof of Lemma 3.4.

Notice that the various conditions of this lemma are properties of $z$ and $M$, and not of the subspace $[M z]$; a priori, condition (1) for $z$ need not imply (1) for an arbitrary vector $x$ such that $[M x]=[M z]$. The hypotheses of Theorem 2 are set to avoid this difficulty.

ThEOREM 2. The following conditions on a $W^{*}$-algebra $M$ on $\mathfrak{S}$ and a vector $z$ in $\mathfrak{S}$ are equivalent:

(A) The projection $E_{[M z]}$ in $M^{\prime}$ is finite;

(B) If $x$ is any vector in $[M z]$ and if $y$ is any vector in $[M x]$, then $y$ is integrable $(M, x)$;

(C) If $x$ is any vector in $[M z]$, and if $\rho$ is any $C A$ state of $M^{\prime}$ in the relation $\rho<\omega_{x}\left(M^{\prime}\right)$, then there exists a vector $y$ integrable $(M, x)$ such that $\rho=\omega_{y}\left(M^{\prime}\right)$; and each of the dual conditions $\left(\mathrm{A}^{\prime}\right),\left(\mathrm{B}^{\prime}\right)$, and $\left(\mathrm{C}^{\prime}\right)$. 
Proof. The equivalence of (B), (C), $\left(B^{\prime}\right)$, and $\left(C^{\prime}\right)$ follows from Lemma 3.4. In proving the equivalence of $(A)$ and $\left(A^{\prime}\right)$ (a result announced by Kaplansky in [5]), we shall make use of the following key corollary to the BT-Theorem (see $\left[8\right.$, p. 178]): for any vectors $x$ and $y, E_{[M x]} \prec E_{[M y]}\left(M^{\prime}\right)$ implies $E_{\left[M^{\prime} x\right]}$ $\prec E_{\left[M^{\prime} y\right]}(M)$.

First, assume that the condition $\left(1^{\prime}\right)$ of Lemma 3.4 holds and that $E_{\left[M^{\prime} z\right]}$ is infinite. There will exist then a partial isometry $V$ in $M$ and a projection $Q<E_{\left[M^{\prime} z\right]}$ such that $V^{*} V=E_{\left[M^{\prime} z\right]}$ and $V V^{*}=Q$. As is easily seen, $\left[M^{\prime} Q z\right]$ $=\left[M^{\prime} V z\right]$. Using the above corollary to the BT-Theorem, we see this implies that $\left.E_{[M Q z]} \sim E_{[M V z}\right]\left(M^{\prime}\right)$. Since $\left(1^{\prime}\right) \leftrightarrow(1)$ in Lemma 3.4 and since $[M V z]$ $=[M z]$, we must have $E_{[M(Q z)]}<E_{[M z]}$. Therefore, the projection $E_{[M z]}$ in $M^{\prime}$ is infinite also. By duality, if $E_{[M z]}$ is infinite, then so is $E_{\left[M^{\prime} z\right]}$. Hence, assuming condition (1'), $E_{[M z]}$ is finite if and only if $E_{\left[M^{\prime} z\right]}$ is finite. On the other hand, assume that condition $\left(1^{\prime}\right)$ is violated. Then, for some $Q<E_{\left[M^{\prime} z\right]}$, $[M Q z]=[M z]$. As before, this implies that $E_{\left[M^{\prime} z\right]} \sim Q(M)$, so that $E_{\left[M^{\prime} z\right]}$ is infinite. Since (1) is also violated, $E_{[M z]}$ is likewise infinite. From this argument we can draw two conclusions: that conditions $(\mathrm{A})$ and $\left(\mathrm{A}^{\prime}\right)$ are equivalent, and that either of these conditions implies condition (1) of Lemma 3.4. Again by Lemma 3.4, it is easy to conclude that either $(A)$ or $\left(A^{\prime}\right)$ implies (B).

To complete the proof, we must show that (B) implies (A) (or the dual $\left(\mathrm{B}^{\prime}\right)$ implies $\left.\left(\mathrm{A}^{\prime}\right)\right)$. We shall do this by assuming that the projection $E_{\left[M^{\prime} z\right]}$ is infinite and that $\left(B^{\prime}\right)$ holds and showing that these assumptions together lead to a contradiction. In what follows, we shall write $E_{\left[M^{\prime} z\right]}=R$ and denote by $V$ and $S$ respectively a partial isometry in $M$ and a projection in $M$ such that $V^{*} V=R$ and $V V^{*}=S<R$.

Consider the three subspaces $[y \in \mathfrak{S} \mid((I-V) x, y)=0$, for all $x \in \mathfrak{S}]$, $\left[y \in \mathfrak{S} \mid y=V^{*} y\right]$, and $[y \in \mathfrak{S} \mid y=V y]$. We assert that these subspaces coincide. In fact, $[y \mid((I-V) x, y)=0]=\left[y \mid y=V^{*} y\right] \subseteq[y \mid R y=y$ and $V y=S y]$. Now, if $y$ belongs to the latter set, then $\|S y\|^{2}=\|V y\|^{2}=\|y\|^{2}=\|(I-S) y\|^{2}$ $+\|S y\|^{2}$, so $(I-S) y=0$ and $V y=S y=y$. Therefore, this set is contained in $[y \mid y=V y]$. But, in turn, it is easy to see that $[y \mid y=V \cdot y] \subseteq\left[y \mid y=V^{*} y\right]$. The assertion is proved.

The projection $F$ on $[y \mid V y=y]$ is in $M$, since this subspace reduces $M^{\prime}$. It is easy to see that $(V-F)$ is a partial isometry in $M$ with initial domain $(R-F) \mathfrak{S}$ and terminal domain $(S-F) \mathfrak{S}$. Moreover $(V-F)$ has no fix points. Therefore, if $V$ has fix points, we can replace the projection $R$ by the projection $E_{\left[M^{\prime}(R-F) z\right]}$, the projection $S$ by the projection $S-F$, and the partial isometry $V$ by the partial isometry $V-F$-this, without altering our assumption, $\left(\mathrm{B}^{\prime}\right)$ and not $\left(\mathrm{A}^{\prime}\right)$. Accordingly, we shall assume that $V$ has no fix points.

In this event, $(I-V)$ has dense range in $\mathfrak{T}$, for $[y \mid((I-V) x, y)=0$, for all $x$ in $\mathfrak{S}]=0$. There exists, therefore, a closed symmetric operator $H$ in 
$C(M)$ of which $V$ is the Cayley transform; one has $H=i(I+V)(I-V)^{-1}$, and Domain $H=$ Range $(I-V)$. So the vector $x=(I-V) z$ belongs to Domain $H$. We show next that $\left[M^{\prime} x\right]=\left[M^{\prime} z\right]$ and that (Domain $\left.H\right) \cap\left[M^{\prime}(R-S) x\right]=0$. In fact, if $y \in\left[M^{\prime} z\right] \ominus\left[M^{\prime} x\right]$, then $\left(\left(I-V^{*}\right) y, M^{\prime} z\right)=0$, so $y=V^{*} y$; because $V$ (and therefore $V^{*}$ ) has no fix points, this means that $y=0$. Also, if $(I-V) y \in($ Domain $H) \cap\left[M^{\prime}(R-S) z\right]$, then $(I-R) y=0$ and $(I-V) y$ $=(R-S)(I-V) y=(I-V) y-S(I-V) y$, so that $S y=V y$. As we have seen above, this implies that $y=V y$, so then $y=0$.

We are now in a position to get a contradiction. Since $\left(\mathbf{B}^{\prime}\right)$ holds, so must condition $\left(2^{\prime}\right)$ of Lemma 3.4 hold for the vector $x$. Now, the proof of the implication (2) $\rightarrow(3)$ in Lemma 3.4 gave rise to the following fact: if $P$ is any projection in $M$ less than $E_{\left[M^{\prime} x\right]}$, and if $T$ is an element of $C(M)$ whose domain contains $x$, then (Domain $T) \cap\left[M^{\prime} P x\right]$ is dense in $\left[M^{\prime} P x\right]$. In the present case, we take for $P$ the projection $R-S$ and for $T$ the operator $H$. By the preceding paragraph, (Domain $H) \cap\left[M^{\prime}(R-S) x\right]=0$, a contradiction. Theorem 2 is proved.

Notice that, in condition (C) of Theorem 2, the vector $y$ such that $\rho=\omega_{y}\left(M^{\prime}\right)$ can be chosen alternatively as a vector integrable $\left(M^{\prime}, x\right)$; this fact follows readily from $\left(\mathrm{B}^{\prime}\right)$ and Lemma 3.1 .

Theorem 2 puts a definite limitation on the class of $W^{*}$-algebras for which the Radon-Nikodým theorem will hold without further restriction on the CA states involved-this, as long as one insists on having closed operators for the Radon-Nikodým derivatives. As we see, the amenable $W^{*}$-algebras are those in which all cyclic projections are finite. Unfortunately, besides purely infinite rings, this excludes even certain factors in the pairing $\left(\mathrm{II}_{\infty}, \mathrm{II}_{\infty}\right)$. A partial Radon-Nikodým theorem does hold in the cyclic case, however, for the class of $W^{*}$-algebras containing no purely infinite projections; by dropping integrability conditions, we prove in the corollary to follow that at least the topological part of the Radon-Nikodým theorem remains valid for such algebras.

Corollary 3.1. Let $M$ be a $\sigma$-finite $W^{*}$-algebra on $\mathfrak{W}$. There exist in $M$ two orthogonal central projections $N_{e}$ and $N_{i}$ with the properties that $N_{e}+N_{i}=I, N_{i}$ is (maximal) purely infinite, and each projection $P$ in $M$ less than $N_{e}$ is the union of an increasing sequence of finite projections. Moreover, if $x$ is any vector such that $N_{i} x=0$, and if $\rho$ is any $C A$ state of $M$ in the relation $\rho<\omega_{x}(M)$, then there exists a vector $y$ in $\left[M^{\prime} x\right] \cap[M x]$ such that $\rho=\omega_{y}(M)$.

Proof. We call two projections $P$ and $Q$ in $M$ parallel, and write $P \| Q$, in case there exists a central projection separating $P$ and $Q-$ that is, a projection $N$ in $M \cap M^{\prime}$ such that $N P=P$ and $N Q=0$. Given any two projections $P$ and $Q$ in $M$, according to a theorem of Dixmier [3, Theorem 6] (which was, effectively, first proved by Maeda [7]), there exists a central projection $N$ such that $P N$ is equivalent to part of $Q N \bmod M$, and $(I-N) Q$ is equivalent 
to part of $(I-N) P \bmod M$. In other words, if two projections are not parallel, then part of one is equivalent to (a nonzero) part of the other.

If a projection $Q$ is purely infinite, then so is the minimal central projection $N_{Q}$ with the property that $N_{Q} Q=Q$. In fact, it is obvious by the Dixmier criterion that any finite projection $P$ in $M$ will necessarily satisfy $P \| Q$. If $N_{Q}$ were not purely infinite, there would exist by definition a nonzero finite $P \leqq N_{Q}$. This and the fact that $P \| Q$ would contradict the minimality of $N_{\mathbf{Q}}$.

Assume that there exist purely infinite projections in $M$. It follows that there exist purely infinite central projections. Let $\left\{N_{n}\right\}$ be a maximal sequence of mutually orthogonal purely infinite central projections $(\neq 0)$, and let $N_{i}=\sum_{n} N_{n}$. The projection $N_{i}$ is purely infinite, for if $P$ is any finite projection satisfying $P \leqq N$, then necessarily $P N_{n}=0$ for all $n$, so that $P=\sum_{n} P N_{n}=0$. We assert that $N_{i}$ is actually the maximal purely infinite projection in $M$; in other words, we assert that $N_{i} Q=Q$, for any purely infinite projection $Q$ in $M$. To prove this, it suffices to show that $N_{i} N_{Q}=N_{Q}$, with $N_{Q}$ defined as above. But it is easy to see that $N_{Q}-N_{Q} N_{i}$ is either 0 or purely infinite. The last possibility is ruled out by the maximality of $N_{i}$. Therefore, a unique maximal purely infinite projection exists, and this projection is central.

Define $N_{e}=I-N_{i}$, and let $P$ be any projection in $M$ satisfying $P \leqq N_{e}$. It follows by construction that there exists a finite projection $P_{1} \leqq P$. By exhaustion, there exists a sequence $\left\{P_{n}\right\}$ of mutually orthogonal finite projections in $M$ such that $P=\sum_{n} P_{n}$. By a theorem of Kaplansky [6, Theorem 6.2], the (lattice) union of a finite number of finite projections in any $W^{*}$-algebra is itself finite. Therefore, $\sum_{i=1}^{n} P_{i}$ is an increasing sequence of finite projections in $M$ whose union is $P$. The first part of the corollary is proved.

We consider now a vector $x$ such that $N_{i} x=0$ and a CA state $\rho$ of $M$ in the relation $\rho<\omega_{x}(M)$. It follows that $N_{e} E_{\left[M^{\prime} x\right]}=E_{\left[M^{\prime} x\right]}$, so we may choose an increasing sequence $\left\{P_{n}\right\}$ of finite projections in $M$ whose LUB is $E_{\left[M^{\prime} x\right]}$. It is clear that $\rho_{P_{n}}<\omega_{P_{n} x}(M)$. Therefore, by Theorem 2 (and the remark following Theorem 2), there exist vectors $y_{n}$ in $\left[M P_{n} x\right]$ such that $\rho_{P_{n}}$ $=\omega_{y_{n}}(M)$. In order to show that $\rho=\omega_{y}(M)$, for some vector in $y[M x]$ (and necessarily in $\left.\left[M^{\prime} x\right]\right)$, it will suffice to prove that a cauchy sequence $\left\{z_{n}\right\}$ exists with the properties that $\omega_{z_{n}}=\omega_{y_{n}}(M)$ and $z_{n} \in[M x]$; the limit $y$ of such a sequence will trivially meet our requirements.

We construct such a sequence by induction. Put $z_{1}=y_{1}$, and suppose that $z_{i}$ is defined for $i \leqq n$ and satisfies the conditions $\omega_{z_{i}}=\omega_{y_{i}}(M), z_{i} \in[M x]$, and $P_{j} z_{i}=z_{j}$ whenever $i \geqq j$. We define $z_{n+1}$ as follows: by definition of the $y_{n}$, $\omega_{P_{n} y_{n+1}}=\omega_{y_{n}}=\omega_{z_{n}}(M)$; as in Lemma 3.1, it follows that there exists a partial isometry $V^{\prime}$ in $M^{\prime}$ with initial domain $\left[M P_{n} y_{n+1}\right]$ and terminal domain $\left[M z_{n}\right]$ such that $V^{\prime}\left(P_{n} y_{n+1}\right)=z_{n}$; now, the projection $R=E_{\left[M v_{n+1}\right]} \bigvee E_{\left[M z_{n}\right]}$ is finite, being the union of two finite projections, and the projections $E_{\left[M P_{n} y_{n+1}\right]}$ and $E_{\left[M z_{n}\right]}$ are equivalent $\bmod M$ and less than $R$; by a well known property of 
finite projections, it follows that the projections $R-E_{\left[M P_{n} y_{n+1}\right]}$ and $R-E_{\left[M z_{n}\right]}$ are likewise equivalent $\bmod M$; on these grounds, we can enlarge the initial domain of the partial isometry $V^{\prime}$ defined above so that $V^{\prime}$ will satisfy the additional condition $\left(V^{\prime}\right)^{*} V^{\prime}=V^{\prime}\left(V^{\prime}\right)^{*}=R$; this being done, we set $z_{n+1}$ $=V^{\prime} y_{n+1}$; it results that $\omega_{z_{n+1}}=\omega_{V^{\prime} y_{n+1}}=\omega_{y_{n+1}}(M)$ (since $\left(V^{\prime}\right)^{*} V^{\prime}=R$ ) and that $P_{n} z_{n+1}=V^{\prime}\left(P_{n} y_{n+1}\right)=z_{n}$; this completes the construction. To conclude, we shall show that the sequence $\left\{z_{n}\right\}$ so defined is cauchy. In fact, if $n \leqq m$, then $\left\|z_{n}-z_{m}\right\|^{2}=\left(z_{n}, z_{n}\right)+\left(z_{m}, z_{m}\right)-\left(P_{n} z_{m}, z_{n}\right)-\left(z_{n}, P_{n} z_{m}\right)=\left(z_{m}, z_{m}\right)-\left(z_{n}, z_{n}\right)$ $=\rho\left(P_{m}\right)-\rho\left(P_{n}\right)$, which tends to 0 with $n$ and $m$. This proves the corollary.

4. A structure theorem for finite rings. The identity $I$ in a $\sigma$-finite finite $W^{*}$-algebra $M$ can be expressed in many ways as a sum $\sum_{n} E_{\left[M^{\prime} x_{n}\right]}$ of mutually orthogonal cyclic projections in $M$. It is a question of some importance in the study of finite $W^{*}$-algebras to determine when the identity can be expressed as a finite sum of cyclic projections. While this property is realized in the "extreme" examples of finite $W^{*}$-algebras, factors, and $\sigma$-finite abelian $W^{*}$-algebras, examples show it is not realized in all finite $W^{*}$-algebras (a fact leading to some topological anomalies, as we shall see in \$5). Precise criteria are developed below for the existence in a finite $W^{*}$-algebra of a finite number of cyclic projections whose union is the identity (see Lemma 4.4). The main result (Theorem 3 ) is a fragment of the multiplicity theory for finite rings: any $\sigma$-finite finite $W^{*}$-algebra is invariantly expressible as a direct sum of finite $W^{*}$-algebras, all central projections in a given summand being the union of the same minimal finite number of cyclic projections. This fact enables us, in later work, to treat these summands as the generic case.

Definition 4.1. A $W^{*}$-algebra $M$ is essentially finite if each cyclic projection in $M$ (that is, each projection of the form $E_{\left[M^{\prime} z\right]}$ ) is finite (in $M$ ).

Using Theorem 2, we see that the commutant $M^{\prime}$ of an essentially finite $W^{*}$-algebra $M$ is itself essentially finite.

Lemma 4.1. Let $R$ be a nonzero $\sigma$-finite projection in an essentially finite $W^{*}$-algebra $M$. Then there exists a cyclic projection $Q$ in $M, Q \leqq R$, which is maximal in the sense that any cyclic projection (in $M$ ) less than $R$ is equivalent to part of $Q \bmod M$.

Proof. We show first that the LUB $P$ of any increasing sequence $\left\{P_{n}\right\}$ of cyclic projections in $M$ and less than $R$ is itself a cyclic projection. In fact, choose vectors $x_{n}$ of norm $1 / 2^{n}$ such that $P_{n}=E_{\left[M^{\prime} x_{n}\right]}$, and define $\rho=\sum_{n} \omega_{x_{n}}$. Plainly, $\rho$ is a CA state of $M$ with the property that its carrier projection $E_{\rho}=P$. Moreover, $\rho_{P_{n}}<\omega_{x_{n}}(M)$, and it follows by Theorem 2 that there exist vectors $y_{n}$ such that $\rho_{P_{n}}=\omega_{y_{n}}(M)$. By the same argument used in the proof of the Corollary 3.1, we see that the $y_{n}$ can be chosen to form a cauchy sequence and, of course, that the limit $y$ of this cauchy sequence will satisfy $\rho=\omega_{y}(M)$. It is obvious that $E_{\left[M^{\prime} y\right]}=E_{\rho}(=P)$, therefore $P$ is cyclic.

We prove next that, given cyclic projections $P$ and $Q$ (each being less than 
$R$ ), there exists a cyclic projection $Q^{\prime}$ in $M$ such that $Q \leqq Q^{\prime} \leqq R$ and $P$ $\prec Q^{\prime}(M)$. By the comparability theorem of Dixmier cited above, there exists a central projection $N$ and a projection $P_{1} \leqq P$ such that $N P \prec N Q(M)$ and $(I-N) P_{1}=P_{1} \sim(I-N) Q(M)$. According to the latter equivalence, there exists a partial isometry $V$ in $M$ such that $V P_{1} V^{*}=(I-N) Q$. By a method analogous to that used in Corollary 3.1, we see that $V$ can be so chosen that $V^{*} V=V V^{*}=(I-N) P$. After this alteration, we let $Q^{\prime}=N Q+V(I-N) P V^{*}$. Trivially, $Q \leqq Q^{\prime} \leqq R$ and $P<Q^{\prime}(M)$. That $Q^{\prime}$ is cyclic follows from the fact that the sum of any two parallel cyclic projections is itself cyclic.

Finally, we construct a maximal cyclic projection $\leqq R$ by transfinite induction. Let $\left\{P_{\alpha}\right\}_{\alpha<r}$ be a well-ordering of nonzero cyclic projections $\leqq R$ (obviously such exist). Define $Q_{1}=P_{1}$, and suppose that $Q_{\alpha}$ is defined for $\alpha<\theta<\tau$ with the properties that $P_{\alpha}<Q_{\beta}(M)$ and $Q_{\alpha} \leqq Q_{\beta} \leqq R$, whenever $\alpha \leqq \beta<\theta$. Let $Q_{\theta}^{\prime}=\mathrm{V}_{\alpha<\theta} Q_{\alpha}$. By $\sigma$-finiteness, this union can be replaced by a countable union, and so by the first paragraph $Q_{\theta}^{\prime}$ is cyclic. By the second paragraph, there exists a cyclic projection $Q_{\theta}$ such that $Q_{\theta}^{\prime} \leqq Q_{\theta} \leqq R$ and $P_{\theta}$ $<Q_{\theta}(M)$. This defines $Q_{\theta}$. Let $Q=\mathrm{V}_{\alpha<r} Q_{\alpha}$. Again by the first paragraph, it follows that $Q$ is a cyclic projection $\leqq R$. Moreover, by construction, if $P$ is any cyclic projection $\leqq R$, then $P \prec Q(M)$. This proves the lemma.

Definition 4.2. By a cyclic dissection of the identity (operator) $I$ in a $W^{*}$-algebra $M$ we shall mean a (finite or countable) sequence $\left\{P_{n}\right\}$ of nonzero mutually orthogonal cyclic projections in $M$ with the properties that $I=\sum_{n} P_{n}$ and $P_{n}$ is maximal cyclic (in the sense of Lemma 4.1) in $\left(I-\sum_{i=1}^{n-1} P_{i}\right)$, for $n=2,3, \cdots$, and $P_{1}$ is maximal cyclic in $M$. Two such dissections $\left\{P_{n}\right\}$ and $\left\{Q_{n}\right\}$ of the identity in $M$ are called unitarily equivalent if there exists a unitary operator $U$ in $M$ such that $U P_{n} U^{*}=Q_{n}$, for all $n$. The length of such a dissection is the number (finite or $\infty$ ) of $P_{n}$ involved.

Lemma 4.2. Any $\sigma$-finite essentially finite $W^{*}$-algebra $M$ has a cyclic dissection of the identity in $M$, and any two such dissections are unitarily equivalent (and have, therefore, the same length).

Proof. The existence of such a dissection follows from Lemma 4.1 and an obvious exhaustion argument. Suppose that $\left\{P_{n}\right\}$ and $\left\{Q_{n}\right\}$ determine two such dissections of $I$ in $M$. We shall prove there exists a unitary $U$ in $M$ such that $U P_{n} U^{*}=Q_{n}$, for all $n$.

The projections $P_{1}$ and $Q_{1}$ must be equivalent, since each is maximal cyclic. Therefore, there exists a partial isometry $V_{1}$ in $M$ such that $V_{1}^{*} V_{1}=P_{1}$ and $V_{1} V_{1}^{*}=Q_{1}$. Now, if either $P_{2}$ or $Q_{2}$ is 0 , then both are 0 , for otherwise $M$ would contain an infinite cyclic projection. So we may suppose both are nonzero. Again by [6, Theorem 6.2], the projection $R=\left(P_{1}+P_{2}\right) \vee Q_{1}$ is finite. As before, $R-P_{1} \sim R-Q_{1}(M)$. This means that $P_{2}$ is equivalent to part of $\left(I-Q_{1}\right) \bmod M$. Since this part of $\left(I-Q_{1}\right)$ is then cyclic, and since $Q_{2}$ is maximal cyclic in $\left(I-Q_{1}\right), P_{2}$ is equivalent to part of $Q_{2}$. By symmetry 
(and the analogue of the Schroeder-Bernstein theorem cited earlier), $P_{\mathbf{2}}$ is equivalent to $Q_{2}$, and so there exists a partial isometry $V_{2}$ in $M$ such that $V_{2}^{*} V_{2}=P_{2}$ and $V_{2} V_{2}^{*}=Q_{2}$. Continuing inductively, one constructs for each $n$ such that $P_{n} \neq 0$ a partial isometry $V_{n}$ in $M$ with $V_{n}^{*} V_{n}=P_{n}$ and $V_{n} V_{n}^{*}=Q_{n}$. The operator $U=\sum_{n} V_{n}$ is obviously unitary, and it meets our requirements.

The proof of Lemma 4.2 shows, in fact, that any projection $P$ in $M$ has a cyclic dissection, determined up to unitary equivalence.

Lemma 4.3. Let $\left\{P_{i}\right\}$ be a finite or countable set of projections determining a cyclic dissection of $I$ in a $W^{*}$-algebra $M$, and let $N_{n}$ be the maximal central projection $\leqq \sum_{i=1}^{n} P_{i}$. Then, for each $n$, the projections $\left(I-N_{n}\right) P_{1}, \cdots$, $\left(I-N_{n}\right) P_{n}$ are equivalent $\bmod M$.

Proof. It is obvious, of course, that the projections $N_{n}$ exist, and plainly $N_{1} \leqq N_{2} \leqq \cdots \leqq N_{n}$. As the basis of an inductive proof, we assume that the projections $\left(I-N_{n-1}\right) P_{1}, \cdots,\left(I-N_{n-1}\right) P_{n-1}$ are nonzero and equivalent mod $M$. Since $\left(I-N_{n}\right) \leqq\left(I-N_{n-1}\right)$, it follows also that the projections $\left(I-N_{n}\right) P_{1}, \cdots,\left(I-N_{n}\right) P_{n-1}$ are also mutually equivalent $\bmod M$. We may assume they are nonzero (that is, $P_{n+1} \neq 0$ ).

Observe first that if $N$ is a central projection and $N P_{n+1}=0$, then $N \leqq N_{n}$. To see this, write $N=N\left(I-\sum_{i=1}^{n} P_{i}\right)+N\left(\sum_{i=1}^{n} P_{i}\right)$, and observe that it suffices to show that $N\left(I-\sum_{i=1}^{n} P_{i}\right)=0$, or what comes to the same, that any cyclic projection $R \leqq N\left(I-\sum_{i=1}^{n} P_{i}\right)$ is necessarily 0 . Now, if $R$ is such a cyclic projection, it follows on the one hand that $R$ is equivalent to part of $P_{n+1}$, by the maximality of $P_{n+1}$ in $\left(I-\sum_{i=1}^{n} P_{i}\right)$, and on the other hand that $R \| P_{n+1}$, since $N R=R$ and $N P_{n+1}=0$. Clearly then $R=0$.

Since $P_{n}$ is equivalent to part of $P_{n-1}$ by definition, there exists a projection $P_{n}^{\prime} \leqq P_{n-1}$ equivalent to $P_{n} \bmod M$. Observe that $\left(P_{n-1}-P_{n}^{\prime}\right) \| P_{m+1}$; in fact, if there exist equivalent projections $R$ and $R^{\prime}, 0<R^{\prime} \leqq\left(P_{n-1}-P_{n}^{\prime}\right)$ and $R \leqq P_{n+1}$, then $P_{n}+R$ is a cyclic projection (being equivalent to $P_{n}^{\prime}+R^{\prime}$ ) which satisfies $P_{n}<\left(P_{n}+R\right) \leqq\left(I-\sum_{i=1}^{n-1} P_{i}\right)$; but this contradicts the maximality of $P_{n}$ in $\left(I-\sum_{i=1}^{n-1} P_{i}\right)$. Now, by construction, $\left(I-N_{n}\right) P_{n-1}$ $>\left(I-N_{n}\right) P_{n}>\left(P_{n+1}\right) \bmod M$. Plainly, $\left(I-N_{n}\right)\left(P_{n-1}-P_{n}^{\prime}\right) \| P_{n+1}$, so there exists a central projection $N^{\prime}$ such that $N^{\prime}\left(I-N_{n}\right)\left(P_{n-1}-P_{n}^{\prime}\right)=\left(I-N_{n}\right)\left(P_{n-1}-P_{n}^{\prime}\right)$ and $N^{\prime} P_{n+1}=0$. It follows by the second paragraph that $N^{\prime} \leqq N_{n}$, and we see then that $\left(I-N_{n}\right)\left(P_{n-1}-P_{n}^{\prime}\right)=0$. Recalling the definition of $P_{n}^{\prime}$, this says that $\left(I-N_{n}\right) P_{n-1}$ is equivalent to $\left(I-N_{n}\right) P_{n} \bmod M$, completing the induction.

Definition 4.3. By the length of the identity $I$ in a $\sigma$-finite essentially finite $W^{*}$-algebra $M$, we mean the length $n=\infty, 1,2, \cdots$ of any cyclic dissection of the identity $I$ in $M$. We say that the identity in $M$ is uniformly of length $n=\infty, 1,2, \cdots$ provided any nonzero central projection $N$ in $M$ has a cyclic dissection of length $n$.

In these terms, as the reader can easily verify, the identity in any finite 
factor is uniformly of length $n$, for some finite $n$, and the identity in any $\sigma$-finite abelian $W^{*}$-algebra is (uniformly) of length 1 .

At this juncture, we derive a minimax criterion which gives explicitly the length of the identity in any $\sigma$-finite finite $W^{*}$-algebra $M$. By a finite dimension function $D$ on $M$ we shall mean a real-valued function defined on the collection $M_{P}$ of projections in $M$, and subject to the following conditions: (i) $0<D(P)<\infty$, for each nonzero $P$ in $M_{P}$; (ii) $D$ is additive on finite sets of mutually orthogonal projections; and (iii) $D$ assumes the same value on projections equivalent $\bmod M$. Any $\sigma$-finite finite $W^{*}$-algebra has finite dimension functions; in fact, if $T(\cdot)$ denotes the center-valued trace on $M$ (see Dixmier [3, Theorem 10]), and if $z$ is a vector so chosen that $E_{\left[M^{\prime} z\right]}$ is maximal cyclic in $M$, then $D(P)=(T(P) z, z)$ is a finite dimension function on $M$, as follows from the properties of $T(\cdot)$ and the fact that $A z=0$ entails $A=0$, for any $A$ in $C=M \cap M^{\prime}$.

Lemмa 4.4. Let $M$ be a $\sigma$-finite finite $W^{*}$-algebra, let $D$ be any finite dimension function on $M$, let $M_{P}^{c}$ denote the collection of cyclic projections in $M$, and let $C_{P}^{0}$ denote the collection of nonzero central projections. Define $c_{M}$ $=\inf _{N \in c_{P}^{0}}\left[\sup _{P \in M_{P}^{c}} D(N P) / D(N)\right]$. Then, $c_{M}=0$ if and only if the length of the identity $I$ in $M$ is $\infty$; and if $c_{M}>0$, the length of $I$ is the least integer $n$ such that $1 / n \leqq c_{M}$. Moreover, the length of $I$ is finite if and only if $T(P)$ has a bounded inverse, for some $P$ in $M_{P}^{c}$.

Proof. First of all, we can simplify the definition of $c_{M}$ by considering only maximal cyclic projections. It is plain, in fact, that if $P$ is any maximal cyclic projection in $M$, then $c_{M}=\inf _{N \in C_{P}^{0}} D(N P) / D(N)$. Now, suppose that the identity in $M$ has a cyclic dissection of length $n<\infty$. If $P_{1}, \cdots, P_{n}$ is such a dissection, we can assume that $P_{1}=P$. Considering the central projections $N_{i}$ defined in Lemma 4.3, we have by that lemma the equivalences $\left(I-N_{n-1}\right) P_{1} \sim \cdots \sim\left(I-N_{n-1}\right) P_{n-1}(M)$. Let $N=I-N_{n-1}$. We have $D\left(N P_{n}\right)$ $\neq 0$, since otherwise it follows from definitions that $P_{n}=0$, and also $D\left(N P_{1}\right)$ $=\cdots=D\left(N P_{n-1}\right)$. Therefore, $D(N)=\sum_{i=1}^{n} D\left(N P_{i}\right)>(n-1) D(N P)$, and consequently $c_{M} \leqq D(N P) / D(N)<1 /(n-1)$. On the other hand, since $P_{i}$ $\prec P(M)$ for each $i$, we have $D\left(N P_{i}\right) \leqq D(N P)$, for each $i$ and each $N$ in $C_{P}^{0}$. Therefore, $D(N) \leqq n D(N P)$, for each $N$ in $C_{P}^{0}$, and consequently $c_{M} \geqq 1 / n$. Now, if the identity has length $\infty$, we see by the above reasoning that $c_{M}<1 / n$, for each integer $n$, so that $c_{M}=0$; and conversely, if $c_{M}=0$, it follows by what we have already proved that $I$ cannot have finite length. It remains to establish that the length of $I$ is finite if and only if $T(P)$ has a bounded inverse, for some cyclic projection $P$ in $M$, or what comes to the same, if and only if $T(P)$ has a bounded inverse, for some maximal cyclic projection $P$ in $M$.

Choose a vector $z$ such that $P=E_{\left[M^{\prime} z\right]}$ is maximal cyclic in $M$. As we have observed above, the function $D(Q)=(T(Q) z, z)\left(Q\right.$ in $\left.M_{P}\right)$ is a finite 
dimension function on $M$. By the first paragraph, the length of $I$ is finite if and only if there exists a constant $K$ such that $D(N) \leqq K D(N P)$, for all $N$ in $C_{P}$, or equivalently, if and only if $\omega_{z} \ll \omega_{(T(P)) 1 / 2_{z}}(C)$. We shall prove that $T(P)$ has a bounded inverse if and only if the latter relation holds.

On the one hand, if $T(P)$ has a bounded inverse, then so does $(T(P))^{1 / 2}$, and it is obvious that $\omega_{z} \ll \omega_{(T(P)) 1 / z_{z}}(C)$. Conversely, suppose that $\omega_{z}$

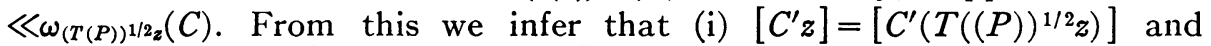
(ii) $\left\{A_{n}\left((T(P))^{1 / 2} z\right)\right\}$ cauchy implies $\left\{A_{n} z\right\}$ cauchy, for any sequence $\left\{A_{n}\right\}$ in $C$. Since already $\left[C\left((T(P))^{1 / 2} z\right)\right] \subseteq[C z]$, we conclude that $\left[C\left((T(P))^{1 / 2} z\right)\right]$ $=[C z]$, by applying (i) and the corollary to the BT-Theorem cited in the first paragraph of Theorem 2. Moreover, by (ii), we see that, for any $x$ in $[C z]$, there exists a $y$ such that $(T(P))^{1 / 2} y=x$-or, in other words, that Range $(T(P))^{1 / 2}$ contains $\left[C_{z}\right]$. Since $D\left(I-E_{\left[C^{\prime} z\right]}\right)=0$, we have $E_{\left[C^{\prime} z\right]}=I$, so that $E_{\left[C^{\prime} z\right]}$ is certainly maximal cyclic in $C$. Again by the corollary to the BT-Theorem, we see that $E_{\left[c_{z}\right]}$ is maximal cyclic in $M^{\prime}$. Therefore, given any vector $y$, there exists a partial isometry $V$ in $C^{\prime}$ such that $V y \in[C z]$ and $V^{*} V y=y$. Since $[C z] \subseteq$ Range $(T(P))^{1 / 2}$, and since Range $(T(P))^{1 / 2}$ reduces $C^{\prime}$, we conclude that $y=V^{*} V y \in$ Range $(T(P))^{1 / 2}$. Therefore, the operator $\left((T(P))^{1 / 2}\right)^{-1}=\left((T(P))^{-1}\right)^{1 / 2}$ is everywhere-definedSA, and hence bounded. Therefore, $T(P)^{-1}$ is bounded, and the proof is completed.

Another easily-deduced criterion is the following: If the identity $I$ in a finite $W^{*}$-algebra $M$ can be expressed in any way as a union of $n<\infty$ cyclic projections, then the length of $I$ is at most $n$. It is important to bear in mind that there exist finite $W^{*}$-algebras whose identities have length $\infty$; a simple example is the ring $M$ formed by taking the direct sum of the full matrix algebras $M_{n}(n=1,2, \cdots)$ on $n$-dimensional unitary space.

Theorem 3. $A$-finite essentially finite $W^{*}$-algebra $M$ contains one and only one set of mutually orthogonal central projections $L_{n}, n=\infty, 1,2, \cdots$, having the properties that $I=\sum_{n} L_{n}$ and that $L_{n}$ is either 0 or uniformly of length $n$. The ring $M$ is finite if and only if $L_{\infty}=0$.

Proof. We prove first that such a set $\left\{L_{n}\right\}$ exists. Let $\left\{P_{n}\right\}$ be any cyclic dissection of the identity in $M$, and let $\left\{N_{n}\right\}$ be the central projections defined in Lemma 4.3. Define $N_{\infty}=\mathrm{LUB}_{n} N_{n}$, and set $L_{\infty}=I-N_{\infty}$. Assuming that $L_{\infty} \neq 0$, we shall show that it is uniformly of length $\infty$. Now, the projections $\left\{L_{\infty} P_{n}\right\}$ form a cyclic dissection of $L_{\infty}$, as is easily checked, and the assertion in question will follow if we prove that these projections are mutually equivalent $\bmod M$. But, for each $n, L_{\infty} \leqq\left(I-N_{n}\right)$, and since $\left(I-N_{n}\right) P_{1} \sim \cdots \sim\left(I-N_{n}\right) P_{n}(M)$, we have $L_{\infty} P_{1} \sim \cdots \sim L_{\infty} P_{n}(M)$, proving the assertion. Next, define $L_{1}=N_{1}$ and $L_{n}=N_{n}-N_{n-1}$, for $n \geqq 2$. By definition, $I=L_{\infty}+\sum_{n<\infty} L_{n}$. Moreover, $L_{n}$ is either 0 or uniformly of length $n$; in fact, by definition of a cyclic dissection of $I$, if $N$ is any central projection, then $N P_{i} \neq 0$ implies $N P_{j} \neq 0$, for all $j \leqq i$; in particular, if $0<N \leqq L_{n}$, then 
$N P_{i} \neq 0$, for $i=1, \cdots, n$, and $N P_{i}=0$ for $i>n$, because $N P_{n}=0$ implies $N \leqq N_{n-1}$, or $N=N\left(N_{n}-N_{n-1}\right)=0$. The existence of a set $\left\{L_{n}\right\}$ is therefore established.

Let $\left\{R_{n}\right\}$ be any other set of mutually orthogonal central projections with the properties that $I=\sum_{n} R_{n}$ and $R_{n}$ is either 0 or uniformly of length $n$. We shall prove that $R_{n}=L_{n}$, for $n=\infty, 1,2, \cdots$. Now, for each $n<\infty$, $R_{n} N_{n-1}$ is a central projection $\leqq R_{n}$ of length $<n$; therefore, $R_{n} N_{n-1}=0$. But, as above, $R_{n} P_{i}=0$ for $i>n$, so $R_{n} \leqq N_{n}$. In other words, $R_{n} \leqq L_{n}$, for $n<\infty$. By the same token, $R_{\infty} N_{n}=0$, for all $n<\infty$, so $R_{\infty} N_{\infty}=0$ and $R_{\infty} \leqq L_{\infty}$. Summarizing these facts, $L_{n} R_{m}=R_{m}$ if $n=m$, and $=0$ otherwise. This and the equality $\sum_{n} L_{n}=\sum_{n} R_{n}$ together imply the conclusion, $R_{n}=L_{n}$, for $n=\infty, 1,2, \cdots$.

For each $n<\infty$, the projection $L_{n}$ is finite, being a (finite) sum of the finite projections $L_{n} P_{1}, \cdots, L_{n} P_{n}$. Since a countable sum of parallel finite projections is finite, we conclude that $N_{\infty}=\sum_{n<\infty} L_{n}$ is finite. Therefore, if $L_{\infty}=\left(I-N_{\infty}\right)=0, M$ is finite. On the other hand, we have seen that $L_{\infty}$ is expressible as a countable sum of mutually orthogonal mutually equivalent projections. Since a nonzero projection of this sort is necessarily infinite, we must have $L_{\infty}=0$ whenever $M$ is finite. The theorem is proved.

An alternative formulation of Theorem 3 is the following: a $\sigma$-finite finite $W^{*}$-algebra can be expressed in one and only one way as a central direct sum of finite $W^{*}$-algebras $M_{n}, n=1,2, \cdots, M_{n}$ being either null or with identity uniformly of (finite) length $n$. (In fact $M_{n}=L_{n} M$ on $L_{n} \mathfrak{S}$ serves as the $n$th summand.)

5. The Radon-Nikodým theorem. In this section, we prove the main theorem announced in the introduction together with a number of topological corollaries. Whereas the trace has entered only incidentally in our work heretofore, it will play a fundamental part in the global studies below-particularly, in establishing the algebraic structure of $C(M)$.

Let $M$ be a fixed $\sigma$-finite finite $W^{*}$-algebra on the hilbert space $\mathfrak{S}$. We shall devote the following series of propositions to a constructive definition of the $L_{2}$-system of a CA state of $M$.

(5.1) If $S$ and $T \in C(M)$, and if $T \supseteq S$, then $T=S$.

(5.2) The sum of two elements (or even a countable number of elements) of $C(M)$ has a unique closed extension in $C(M)$, and $C(M)$ becomes a linear space (over the complex numbers) when addition is defined by taking the closure of sums.

(5.3) The product of two elements of $C(M)$ has a unique closed extension in $C(M)$. With this multiplication, and with the linear operations of (5.2), $C(M)$ is an algebra.

These important facts are proved by Murray and von Neumann [8, pp. 221-229] for finite factors. When we observe that any $\sigma$-finite finite $W^{*}$-algebra has a finite dimension function, in the sense expressed in $\$ 4$, and that complements of equivalent projections are equivalent in any finite $W^{*}$-alge- 
bra, the proofs given in [8] apply almost without change to any $\sigma$-finite finite $W^{*}$-algebra. Accordingly, we omit further proof.

Let $\left\{x_{n}\right\}$ be any sequence of vectors in $\mathfrak{S}$ (of which all but a finite number may be 0 ), subject to the condition $\sum_{n}\left\|x_{n}\right\|^{2}<\infty$, and let $E$ denote the projection $\bigvee_{i} E_{\left[M^{\prime} x_{i}\right]}$; in other words, $E$ is the carrier projection of the state $\sum_{n} \omega_{x_{i}}$ of $M$. We define

$$
\begin{gathered}
S\left[M, E,\left\{x_{n}\right\}\right]=\left[T \text { in } C(M) \mid T E=T, \text { all the } x_{n} \in \text { Domain } T,\right. \text { and } \\
\left.\sum_{n}\left\|T x_{n}\right\|^{2}<\infty\right],
\end{gathered}
$$

and moreover, if $S$ and $T$ are elements of $S\left[M, E,\left\{x_{n}\right\}\right]$, we define

$$
[S, T]=\sum_{n}\left(S x_{n}, T x_{n}\right) .
$$

(5.4) $S\left[M, E,\left\{x_{n}\right\}\right]$ is a linear space (under the linear operations of (5.2)), and the bilinear form $[S, T]$ is an inner product on $S\left[M, E,\left\{x_{n}\right\}\right]$.

(5.5) If $\sum_{m} \omega_{y_{m}}=\sum_{n} \omega_{x_{n}}(M)$, and if $S\left[M, E^{\prime},\left\{y_{m}\right\}\right],[S, T]^{\prime}$ denotes the corresponding system, then $E=E^{\prime}, S\left[M, E,\left\{y_{m}\right\}\right]=S\left[M, E,\left\{x_{n}\right\}\right]$, and $[S, T]^{\prime}=[S, T]$.

The proposition (5.4) is an obvious consequence of (5.2). Proposition (5.5) stems from the following corollary to the spectral theorem, which will be used frequently below: given $T$ in $C(M)$, there exists a sequence $\left\{T_{n}\right\}$ in $M$ with the property that a vector $x$ belongs to Domain $T$ if and only if $\lim _{n}\left\|T_{n} x\right\|^{2}$ exists (and is finite); and in case this limit exists, it equals $\|T x\|^{2}$.

We shall assume in the propositions (5.6)-(5.9) to follow that the identity $I$ in $M$ has finite length.

(5.6) Given any $C A$ state $\rho$ of $M$, there exist vectors $\left\{x_{i}\right\},\left\{y_{i}\right\}$ $(i=1, \cdots, n)$, such that the projections $E_{\left[M^{\prime} x_{i}\right]}$ are mutually orthogonal, $E_{\left[M y_{i}\right]}$ $=E_{\left[M x_{i}\right]}, \bigvee_{i} E_{\left[M^{\prime} y_{i}\right]}=\bigvee_{i} E_{\left[M^{\prime} x_{i}\right]}$, and $\rho=\sum_{i=1}^{n} \omega_{y_{i}}(M)$.

To prove this, suppose first that we have found vectors $x_{1}, \cdots, x_{n}$ giving rise to the relation $\rho \ll \sum_{i=1}^{n} \omega_{x_{i}}(M)$. In these circumstances, we shall show that there exist vectors $y_{1}, \cdots, y_{n}\left(y_{i} \in\left[M x_{i}\right]\right)$ such that $\rho=\sum_{i=1}^{n} \omega_{y_{i}}(M)$. Write $\sigma=\sum_{i=1}^{n} \omega_{x_{i}}(M)$. By a theorem of Segal [13, Theorem 1], there exists a hilbert space $\mathfrak{S}_{\sigma}$, a linear mapping $\eta_{\sigma}$ of $M$ on a dense manifold in $\mathfrak{S}_{\sigma}$, a *-representation $A \rightarrow \phi_{\sigma}(A)$ of $M$ on $\mathfrak{S}_{\sigma}$, and a cyclic vector $\xi_{0}$ for the *-algebra $\phi_{\sigma}(M)$ such that $\phi_{\sigma}(A) \eta_{\sigma}(B)=\eta_{\sigma}(A B)$ and $\sigma(A)=\left(\phi_{\sigma}(A) \xi_{0}, \xi_{0}\right)$, for all $A$ and $B$ in $M$. Since $\rho \ll \sigma(M), \rho$ induces a state of $\phi_{\sigma}(M)$ in the relation $\rho$ $\ll \omega_{\xi_{0}}\left(\phi_{\sigma}(M)\right)$. By Lemma 2.2, there exists a vector $\xi$ in $\mathfrak{S}_{\sigma}$ such that $\rho(A)$ $=\left(\phi_{\sigma}(A) \xi, \xi\right)$, for all $A$ in $M$. Choose a $\sigma$-cauchy sequence $\left\{A_{r}\right\}$ in $M$ such that $\eta_{\sigma}\left(A_{r}\right) \rightarrow \xi$ in $\mathfrak{S}_{\sigma}$, and let $y_{i}=\lim _{r} A_{r} x_{i}$ in $\mathfrak{S}$. It follows that $\rho(A)$ $=\lim _{r}\left(\phi_{\sigma}(A) \eta_{\sigma}\left(A_{r}\right), \eta_{\sigma}\left(A_{r}\right)\right)=\lim _{r} \sum_{i=1}^{n} \omega_{A_{r} x_{i}}(A)=\sum_{i=1}^{n} \omega_{y_{i}}(A)$, for all $A$ in $M$.

Now, in view of Theorem 3 , we can find vectors $z_{1}, \cdots, z_{n}$ such that the 
projections $P_{i}=E_{\left[M^{\prime} z_{i}\right]}$ are mutually orthogonal and satisfy $\sum_{i=1}^{n} P_{i}=I$. It is obvious that $\rho_{P_{i}}<\omega_{z_{i}}(M)$, for each $i$. Therefore, by Theorem 2 , there exists a set of vectors $x_{1}, \cdots, x_{n}\left(x_{i}\right.$ in $\left.\left[M^{\prime} z_{i}\right]\right)$ such that $\rho_{P_{i}}=\omega_{x_{i}}(M)$. Plainly, $\rho \sim \sum_{i=1}^{n} \rho_{P_{i}}(M)$. Also, as was shown in the proof of assertion $\left.{ }^{* *}\right)$ in Lemma $3.4, \rho \ll \sum_{i=1}^{n} \rho_{P_{i}}(M)$. This shows that the conditions of the above paragraph can always be realized. It is easy to see that the vectors $\left\{x_{i}\right\},\left\{y_{i}\right\}$, as defined in our proof, meet all the requirements of (5.6).

Coupled with Theorem 2, proposition (5.6) provides the basis for the proof of the Radon-Nikodým theorem. Incidentally, the space $\mathfrak{S}_{\sigma}$ alluded to above is precisely the space $L_{2}(M, \sigma)$ defined below; it is advantageous for our purposes to take a more concrete approach in defining this notion. We insert now what amounts to a characterization of the kernel of $\phi_{\sigma}$ (see Segal $[14])$.

(5.7) Given a $C A$ state $\rho$ of $M$, define $C_{\rho}=\mathrm{LUB} \mathrm{V}_{i=1}^{n} E_{\left[M x_{i}\right]}$, taken over $\left[\left(x_{1}, \cdots, x_{n}\right) \mid \rho=\sum_{i=1}^{n} \omega_{x_{i}}(M)\right]$. Then $C_{\rho} \in M \cap M^{\prime}, C_{\rho}$ is the GLB of central projections $\geqq E_{\rho}$, and $\rho\left(B^{*} T^{*} T A\right)=0$, for all $A, B$ in $M$ and a fixed $T$ in $M$, if and only if $T C_{\rho}=0$.

If $U^{\prime}$ is any unitary operator in $M^{\prime}$, it is easy to see that $U^{\prime}\left(\bigvee_{i=1}^{n} E_{\left[M x_{i}\right]}\right) U^{\prime *}$ $=\mathrm{V}_{i=1}^{n} E_{\left[M U^{\prime} x_{i}\right]}$. Moreover, if $\rho=\sum_{i=1}^{n} \omega_{x_{i}}(M)$, it is obvious that $\rho$ $=\sum_{i=1}^{n} \omega_{U^{\prime} x_{i}}(M)$ also. We conclude that $U^{\prime} C_{\rho} U^{\prime *} \leqq C_{\rho}$, for all unitary $U^{\prime}$ in $M^{\prime}$. By symmetry, $C_{\rho}$ commutes with all unitary operators in $M^{\prime}$, and consequently $C_{\rho} \in M$. By definition, $C_{\rho} \in M^{\prime}$. Therefore, $C_{\rho}$ is central. Now, since $\rho\left(I-C_{\rho}\right)=0$, we see that $C_{\rho} \geqq E_{\rho}$. Moreover, if $C$ is any central projection satisfying $C_{\rho} \geqq C \geqq E_{\rho}$, and if $\rho=\sum_{i=1}^{n} \omega_{x_{i}}(M)$, then $\left(C_{\rho}-C\right) E_{\left[M x_{i}\right]}=0$, for each $i$. We conclude easily that $\left(C_{\rho}-C\right) C_{\rho}=0$, so $C_{\rho}=C$. The last assertion follows directly from these two characterizations.

(5.8) Let $\left\{x_{i}\right\},\left\{y_{i}\right\}$, and $\left\{z_{i}\right\}(i=1, \cdots, n)$ be three sets of vectors having the following properties: (i) the $E_{\left[M^{\prime} x_{i}\right]}$ are mutually orthogonal; (ii) $\bigvee_{i=1}^{n} E_{\left[M^{\prime} x_{i}\right]}$ $=\mathrm{V}_{i=1}^{n} E_{\left[M^{\prime} y_{i}\right]}$; and (iii) both $E_{\left[M_{i}\right]}$ and $E_{\left[M_{i}\right]} \leqq E_{\left[M x_{i}\right]}$, for all $i$. Then, there exists $a T$ in $C(M)$ such that $T y_{i}=z_{i}$, for all $i$.

First, we exhibit a $T_{1}$ in $C(M)$ such that $T_{1} x_{i}=y_{i}$, for all $i$. To this end, choose for each $i$ an $S_{i}$ in $C(M)$ such that $S_{i} x_{i}=y_{i}$ and $S_{i} E_{\left[M^{\prime} x_{i}\right]}=S_{i}$; such a choice is possible by Theorem 2. Let $T_{1}$ be the closed extension in $C(M)$ of the sum $\sum_{i=1}^{n} S_{i}$ (using (5.2)). By the orthogonality relations (i), $S_{i} x_{j}=0$ if $i \neq j$. Therefore, Domain $\left(\sum_{i=1}^{n} S_{i}\right)$ contains each $x_{j}$, and $T_{1} x_{j}=\left(\sum_{i=1}^{n} S_{i}\right) x_{j}$ $=S_{1} x_{j}=y_{j}$, as desired.

Next, we show there exists a $T_{2}$ in $C(M)$ such that $T_{2} y_{i}=x_{i}$, for each $i$. In fact, by construction and (ii), $E_{\left.\text {[Range } T_{1}\right]} \leqq \bigvee_{i=1}^{n} E_{\left[M^{\prime} y_{i}\right]}=\bigvee_{i=1}^{n} E_{\left[M^{\prime} x_{i}\right]}$. Moreover, [Range $\left.T_{1}\right]$ contains each subspace $\left[M^{\prime} y_{i}\right]$. Therefore, $E_{\left[\text {Range } T_{1}\right]}$ $=\mathrm{V}_{i-1}^{n} E_{\left[M^{\prime} x_{i}\right]}$. If $P$ denotes the projection on $\mathfrak{S} \Theta \sum_{i=1}^{n} \oplus\left[M^{\prime} x_{i}\right]$, it is clear that $\left(T_{1}+P\right) \in C(M)$ coincides with $T_{1}$ on $\sum_{i=1}^{n} \oplus\left[M^{\prime} x_{i}\right]$, and has everywhere dense range in $\mathfrak{G}$. But, an element $T$ in $C(M)$ with dense range always has an inverse in $C(M)$-in fact, in the polar decomposition $T=V H$, we 
can take $V$ unitary, since $M$ is finite; therefore, if $T$ has dense range, so must the SA operator $H$ (in $C(M)$ ); and a SA operator with dense range always has a SA inverse. If we set $T_{2}=\left(T_{1}+P\right)^{-1}$, it follows that $T_{2} y_{i}=x_{i}$, for each $i$, and the assertion is proved.

To conclude, we construct (by the argument of the first paragraph) an operator $T_{3}$ in $C(M)$ such that $T_{3} x_{i}=z_{i}$, for all $i$. Let $T=$ the closed extension in $C(M)$ of $T_{3} T_{2}$ (using (5.3)). The operator $T$ has the desired properties. $[S, T]$.

(5.9) $S\left[M, E, x_{1}, \cdots, x_{n}\right]$ (of (5.4)) is complete in the inner product

Assuming that $\lim _{r, s} \sum_{i=1}^{n}\left\|\left(T_{r}-T_{s}\right) x_{i}\right\|^{2}=0$, for some sequence $\left\{T_{n}\right\}$ in $S\left[M, E, x_{1}, \cdots, x_{n}\right]$, we are to exhibit a $T$ in $S\left[M, E, x_{1}, \cdots, x_{n}\right]$ such that $\lim _{r} \sum_{i=1}^{n}\left\|\left(T_{r}-T\right) x_{i}\right\|^{2}=0$. By (5.6), we can find vectors $\left\{y_{i}\right\},\left\{z_{i}\right\}$ $(i=1, \cdots, m)$, such that the $E_{\left[M^{\prime} z_{i}\right]}$ are mutually orthogonal, $E_{\left[M y_{i}\right]}$ $=E_{\left[M z_{i}\right]}, \bigvee_{i=1}^{m} E_{\left[M^{\prime} y_{i}\right]}=\bigvee_{i=1}^{m} E_{\left[M^{\prime} z_{i}\right]}$, and $\sum_{i=1}^{n} \omega_{x_{i}}=\sum_{i=1}^{m} \omega_{y_{i}}(M)$. Write $w_{i}$ $=\lim _{r} T_{r} y_{i}$. Applying (5.8), with $x_{i}, y_{i}, z_{i}$ replaced by $z_{i}, y_{i}, w_{i}$, we choose a $T$ in $C(M)$ such that $T y_{i}=w_{i}$, for each $i$. Obviously, we may take $T$ in $S\left[M, E, x_{1}, \cdots, x_{n}\right]$. It is plain that $\lim _{r} \sum_{i=1}^{m}\left\|\left(T_{r}-T\right) y_{i}\right\|^{2}=0$, and in view of (5.5), this same relation holds with the $x_{i}$ replacing the $y_{i}$, proving (5.9).

We conclude these preliminary propositions by removing the restriction on the length of the identity in $M$.

(5.10) Let $\rho$ be any $C A$ state of the (arbitrary) $\sigma$-finite finite $W^{*}$-algebra $M$. Then, there exists a sequence of vectors $\left\{x_{n}\right\}$ (of which all but a finite number may be 0$)$ such that $\rho=\sum_{n} \omega_{x_{n}}(M)$. Moreover, the space $S\left[M, E_{\rho},\left\{x_{n}\right\}\right]$ of (5.4) is a hilbert space under the inner product $[S, T]=\sum_{n}\left(S x_{n}, T x_{n}\right)$.

By Theorem 3, we can choose in $M$ a sequence $\left\{L_{i}\right\}$ of mutually orthogonal central projections such that $I=\sum_{i} L_{i}$ and $L_{i}$ is uniformly of (finite) length $i$. We then have $\rho=\sum_{i} \rho_{L_{i}}(M)$. Furthermore, by (5.6), each positive functional $\rho_{L_{i}}$ is expressible as a finite sum of canonical positive functionals (in fact, $i$ such functionals suffice for $\left.\rho_{L_{i}}\right)$. It follows that $\rho$ has a representation $\rho$ $=\sum_{i} \omega_{x_{i}}(M)$ of the sort described. In view of its representation as a finite sum, each nonzero component $\rho_{L i}$ determines a hilbert space, by (5.9). It is trivial that $S\left[M, E_{\rho},\left\{x_{n}\right\}\right]$ in the inner product $[S, T]$ is a direct sum of these component hilbert spaces, and accordingly, is a hilbert space itself.

Definition 5.1. Let $\rho$ be a CA state of the $\sigma$-finite finite $W^{*}$-algebra $M$. By $L_{2}(M, \rho)$, we shall mean the hilbert space $S\left[M, E_{\rho},\left\{x_{n}\right\}\right]$ in the inner product $[S, T]=\sum_{n}\left(S x_{n}, T x_{n}\right),\left\{x_{n}\right\}$ being any sequence of vectors such that $\rho=\sum_{n} \omega_{x_{n}}(M)$.

In view of (5.5), this definition is independent of the particular vectors representing $\rho$. Elements of $L_{2}(M, \rho)$ are intrinsically characterized as those $T$ in $C(M)$ such that $\lim _{n} \rho\left(T_{n}^{*} T_{n}\right)$ exists, $\left\{T_{n}\right\}$ being any sequence of (bounded) operators in $M$ such that a vector $x$ belongs to Domain $T$ if and only if $\lim _{n}\left\|T_{n} x\right\|^{2}$ exists (and equals $\|T x\|^{2}$ ). Given any $T$ in $L_{2}(M, \rho)$, we 
denote by $\rho_{T}$ that positive functional on $M$ defined by the equation $\rho_{T}(A)$ $=[A T, T]$, where $[$,$] denotes the inner product in L_{2}(M, \rho)$.

THeOREM 4. If two $C A$ states $\sigma$ and $\rho$ of the $\sigma$-finite finite $W^{*}$-algebra $M$ are in the relation $\sigma<\rho(M)$, then there exists $a T$ in $L_{2}(M, \rho)$ such that $\sigma=\rho_{T}(M)$.

Proof. By the device of (5.10), we see that it suffices to consider the case in which the identity of $M$ has finite length. With this assumption, then, choose by (5.6) vectors $\left\{x_{i}\right\},\left\{y_{i}\right\}$ such that the projections $E_{\left[M^{\prime} x_{i}\right]}$ are mutually orthogonal, $E_{\left[M y_{i}\right]} \leqq E_{\left[M x_{i}\right]}, \quad \bigvee_{i=1}^{n} E_{\left[M^{\prime} y_{i}\right]}=\bigvee_{i=1}^{n} E_{\left[M^{\prime} x_{i}\right]}$, and $\rho$ $=\sum_{i=1}^{n} \omega_{y_{i}}(M)$. Write $E_{\left[M^{\prime} x_{i}\right]}=P_{i}$. By Corollary 3.1, choose vectors $w_{i}$ in $\left[M^{\prime} x_{i}\right] \cap\left[M x_{i}\right]$ such that $\sigma_{P_{i}}=\omega_{w_{i}}(M)$. As in (5.6), we conclude that there exist vectors $z_{i}$ in $\left[M w_{i}\right]$ such that $\sigma=\sum_{i-1}^{n} \omega_{z_{i}}(M)$. Applying (5.8) (to $x_{i}, y_{i}, z_{i}$, we conclude there exists a $T$ in $C(M)$ such that $T y_{i}=z_{i}$, for all $i$. Since the operator $T E_{\rho}$ also has this latter property, we may assume that $T \in L_{2}(M, \rho)$. Therefore, $\sigma(A)=\sum_{i=1}^{n}\left(A T y_{i}, T y_{i}\right)=\rho_{T}(A)$, for all $A$ in $M$, completing the proof.

As is evident, the Radon-Nikodým derivative $T$ can be chosen to satisfy $T=E_{\rho} T$. Conversely, for any $T$ in $L_{2}(M, \rho), \rho_{E_{\rho} T}<\rho(M)$.

REMARK 5.1. Several criteria are available which enable one to reduce the study of completely additive states of an arbitrary finite $W^{*}$-algebra to that of countably additive states of a $\sigma$-finite finite $W^{*}$-algebra. More explicitly, (1) a finite $W^{*}$-algebra is $\sigma$-finite if and only if its center is $\sigma$-finite, (2) any finite $W^{*}$-algebra is a central direct sum of $\sigma$-finite finite $W^{*}$-algebras, and (3) if $\rho$ is a completely additive state of the finite $W^{*}$-algebra $M$, then $M$ can be expressed as the central direct sum of a $\sigma$-finite finite $W^{*}$-algebra and a finite $W^{*}$-algebra on which $\rho$ vanishes. (As to the proof of these facts, (2) and (3) are immediate corollaries of (1), and (1) follows from the fact that a finite $W^{*}$-algebra $M$ with a $\sigma$-finite center $C$ admits a CA state positive on all nonzero projections-to wit, $(T(A) z, z)$, where $z$ is a cyclic vector for $C^{\prime}$ and $T(\cdot)$ is the center-valued trace on $M$.) Notice, in view of (3), that Theorem 4 holds for any finite $W^{*}$-algebra $M$ when the state $\rho$ of $M$ is assumed to be completely additive.

Theorem 4 assumes a somewhat sharper form when the CA state $\rho$ is unitarily invariant-that is, when $\rho\left(U^{*} A U\right)=\rho(A)$, for all $A$ in $M$ and all unitary operators $U$ in $M$. Here, at least, it is possible to show that the RadonNikodým derivatives can be taken as non-negative SA operators, and that after such a choice they are uniquely determined. The first assertion in the following corollary is a direct generalization of the trace formula for finite factors (see Remark 2.1); the result as such has been announced by I. Kaplansky [5] in a special case (effectively, that in which $M^{\prime}$ has a cyclic vector).

Corollary 5.1. Let $M$ be a $\sigma$-finite finite $W^{*}$-algebra, let $T(\cdot)$ denote the center-valued trace on $M$, and let $\lambda$ be any unitarily invariant $C A$ state of $M$, with $E_{\lambda}=I$. Then, there exists a finite or countable set of vectors $\left\{x_{n}\right\}$ such that 
the projections $E_{\left[M^{\prime} x_{n}\right]}$ determine a cyclic dissection of the identity in $M$ and such that $\lambda(A)=\sum_{n}\left(A x_{n}, x_{n}\right)=\sum_{n}\left(T(A) x_{n}, x_{n}\right)$, for all $A$ in $M$.

Moreover, if $\rho$ is any $C A$ state of $M$, there exists a uniquely determined non-negative $S A$ operator $H$ in $L_{2}(M, \lambda)$ such that $\rho(A)=\lambda(H A H)$ $=\sum_{n}\left(A H x_{n}, H x_{n}\right)$, for all $A$ in $M$.

Proof. Choose vectors $\left\{z_{i}\right\}$ so that the projections $P_{i}=E_{\left[M^{\prime} z_{i}\right]}$ form a cyclic dissection of the identity $I$ in $M$. We have $\lambda_{P_{i}} \sim \omega_{\boldsymbol{z}_{\boldsymbol{i}}}(M)$, and it follows by Theorem 2 that, for appropriate vectors $\left\{x_{i}\right\}, \lambda_{P_{i}}=\omega_{x_{i}}(M)$. Obviously, $E_{\left[M^{\prime} x_{i}\right]}=P_{i}$. Since $\lambda(A B)=\lambda(B A)$, for all $A$ and $B$ in $M$, we have $\lambda(A)$ $=\sum_{i} \lambda\left(A P_{i}\right)=\sum_{i} \lambda\left(P_{i} A P_{i}\right)=\sum_{i} \omega_{x_{i}}(A)$, for all $A$ in $M$.

As is shown by Dixmier [3, Theorem 12], for any $A$ in $M$, the operator $T(A)$ belongs to the uniform closure of the set of all finite convex linear combinations $\sum_{i} \alpha_{i} U_{i} A U_{i}^{*}$, where $U_{i}$ is any unitary operator in $M, \alpha_{i} \geqq 0$, and $\sum_{i} \alpha_{i}=1$. By the unitary invariance of $\lambda, \lambda\left(\sum_{i} \alpha_{i} U_{i} A U_{i}^{*}\right)=\lambda(A)$, for any such linear combination. Therefore, $\lambda(T(A))=\lambda(A)$, for all $A$ in $M$.

In completing the proof, we shall assume (without loss of generality) that the identity $I$ in $M$ is uniformly of (finite) length $n$. Now, given any CA state $\rho$ of $M$, we know by Theorem 4 that $\rho(A)=\sum_{i=1}^{n} \omega_{T x_{i}}(A)$, for some $T$ in $L_{2}(M, \lambda)$. By a simple approximation argument using the unitary invariance of $\lambda$, we see that if $T \in L_{2}(M, \lambda)$, then also $T^{*} \in L_{2}(M, \lambda)$, and moreover $\lambda\left(T^{*} T\right)=\lambda\left(T T^{*}\right)$. This remark and the fact that we can always write a $T$ in $C(M)$ in the form $H U$, where $H$ is non-negative SA in $C(M)$ and $U$ is unitary in $M$, together show that $\rho(A)=\sum_{i=1}^{n}\left(A H x_{i}, H x_{i}\right)$, for all $A$ in $M$ and some non-negative $\mathrm{SA}$ operator $H$ in $L_{2}(M, \lambda)$. (Of course, by (5.5), $H$ is independent of the particular vectors $x_{i}$ used to represent $\lambda$.)

To complete the proof, we must show that this operator $H$ is uniquethat is, that it depends only on the values of $\lambda$ and $\rho$. This will follow if we prove the following assertion: if $H^{\prime}$ is any non-negative SA operator in $L_{2}(M, \lambda)$ such that $\rho(A)=\sum_{i=1}^{n}\left(A H^{\prime} x_{i}, H^{\prime} x_{i}\right)$, for all $A$ in $M$, then $H=H^{\prime}$.

First, we prove that if $T$ and $T^{\prime}$ are any bounded operators in $L_{2}(M, \lambda)$ such that $\lambda\left(T^{*} A T\right)=\lambda\left(T^{*} A T^{\prime}\right)$, for all $A$ in $M$, then $T T^{*}=T^{\prime} T^{*}$. In fact, we have (in the notation of the first paragraph) $\lambda\left(T^{*} A P_{i} B^{*} T\right)$ $=\lambda\left(T^{*} A P_{i} B^{*} T^{\prime}\right)$, for all $i$ and all $A, B$ in $M$. Now $\lambda\left(T^{*} A P_{i} B^{*} T\right)$ $=\lambda\left(B^{*} T T^{*} A P_{i}\right)=\left(T^{*} A x_{i}, T^{*} B x_{i}\right)$, and likewise we have $\lambda\left(T^{*} A P_{i} B^{*} T^{\prime}\right)$ $=\left(T^{*} A x_{i}, T^{*} B x_{i}\right)$. It follows that $\left(T T^{*}-T^{\prime} T^{*}\right) E_{\left[x_{i}\right]}=0$, for all $x_{i}$. This is valid for all vectors $x_{1}, \cdots, x_{n}$ such that $\lambda=\sum_{i=1}^{n} \omega_{x_{i}}(M)$. Therefore, using (5.7), $\left(T T^{*}-T^{\prime} T^{*}\right) C_{\lambda}=0$, and since $C_{\lambda}=I$, this means $T T^{*}=T^{\prime} T^{*}$.

Returning to our problem, let $E(\lambda)$ and $E^{\prime}(\lambda)$ denote the spectral resolutions of $H$ and $H^{\prime}$, respectively. Let $\lambda_{n}$ be an unbounded increasing sequence of real numbers, and let $P_{n}=E\left(\lambda_{n}\right), P_{n}^{\prime}=E^{\prime}\left(\lambda_{n}\right)$. Plainly, $\lim _{n} P_{n}=\lim _{n} P_{n}^{\prime}$ $=I$. Moreover, by the spectral theorem, if we set $H_{n}=P_{n} H$ and $H_{n}^{\prime}=P_{n}^{\prime} H^{\prime}$, then the operators $H_{n}$ and $H_{n}^{\prime}$ are bounded non-negative SA (in $M$ ), a vector $x$ belongs to Domain $H$ (respectively, Domain $H^{\prime}$ ) if and only if the 
sequence $\left\{H_{n} x\right\}$ (respectively, $\left.\left\{H_{n}^{\prime} x\right\}\right)$ is cauchy, and then $H x=\lim _{n} H_{n} x$ $\left(H^{\prime} x=\lim _{n} H_{n}^{\prime} x\right)$. Let $Q_{n}=P_{n} \wedge P_{n}^{\prime}$. The sequence $\left\{Q_{n}\right\}$ plainly increases with $n$. We shall show that $\lim _{n} Q_{n}=I$. In fact, for each $n$, the projections $P_{n} \bigvee P_{n}^{\prime}-P_{n}^{\prime}$ and $P_{n}-P_{n}^{\prime} \wedge P_{n}$ are equivalent $\bmod M$ (see [6, Theorem 5.4]). Therefore, $\lambda\left(P_{n} \vee P_{n}^{\prime}-P_{n}^{\prime}\right)=\lambda\left(P_{n}-P_{n} \wedge P_{n}^{\prime}\right)$, for all $n$. Hence, $\lambda\left(I-P_{n} \wedge P_{n}^{\prime}\right)=1-\lambda\left(P_{n}\right)-\lambda\left(P_{n}^{\prime}\right)+\lambda\left(P_{n} \vee P_{n}^{\prime}\right)$. As $n \rightarrow \infty$, the right-hand side tends to 0 . Therefore, by Lemma $2.3, I-P_{n} \wedge P_{n}^{\prime} \rightarrow 0$ in the strong sequential topology, proving the assertion. (Incidentally, this paragraph provides the basis of proof for (5.2).)

By virtue of our assumption $\sum_{i=1}^{n} \omega_{H x_{i}}=\sum_{i=1}^{n} \omega_{H^{\prime} x_{i}}(M)$ and the facts that $Q_{n} H=Q_{n} H_{n}, Q_{n} H^{\prime}=Q_{n} H_{n}^{\prime}$, we conclude that $\lambda\left(\left(Q_{n} H_{n}\right)^{*} A\left(Q_{n} H_{n}\right)\right)$ $=\lambda\left(\left(Q_{n} H_{n}^{\prime}\right)^{*} A\left(Q_{n} H_{n}^{\prime}\right)\right)$, for all $A$ in $M$. We have proved, under these conditions, that $H_{n} Q_{n} H_{n}=H_{n}^{\prime} Q_{n} H_{n}^{\prime}$, for all $n$. By the preceding paragraph, the manifold $\mathfrak{M}=($ Domain $H) \cap\left(\right.$ Domain $H^{\prime}$ ) is dense in the hilbert space $\mathfrak{S}$ (on which $M$ acts), for $\mathfrak{M}$ contains each subspace $Q_{n} \mathfrak{S}$. Moreover, the contraction of $H$ (or $H^{\prime}$ ) to $\mathfrak{M}$ has a least closed extension, and by (5.1), this closed extension must coincide with $H$ (or $H^{\prime}$ ). Let $x$ and $y$ be any vectors in $\mathfrak{M}$. We have $(H x, H y)=\lim _{n}\left(Q_{n} H_{n} x, H_{n} y\right)=\lim _{n}\left(Q_{n} H_{n}^{\prime} x, H_{n}^{\prime} y\right)=\left(H^{\prime} x, H^{\prime} y\right)$. This quality will hold also when $x$ and $y$ belong to the domain of the least closed extension of the contraction of $H$ to $\mathfrak{M}$, and hence for all $x$ and $y$ in Domain $H$. We see, by symmetry, that Domain $H=$ Domain $H^{\prime}$, and in fact, that the operators $H$ and $H^{\prime}$ are metrically equivalent. But by the spectral theorem (in particular, by the uniqueness of square roots), two metrically equivalent non-negative SA operators on a hilbert space always coincide. Therefore, $H=H^{\prime}$, completing the uniqueness proof.

Recall that the strongest topology on a $W^{*}$-algebra $M$ is that specified by general neighborhoods of 0 of the form $\left[A\right.$ in $M \mid \sum_{i=1}^{\infty}\left\|A x_{i}\right\|^{2}<1$, where $\left\{x_{i}\right\}$ is any sequence of vectors such that $\left.\sum_{i=1}^{\infty}\left\|x_{i}\right\|^{2}<\infty\right]$.

COROLlARY 5.2. The strong and the strongest topologies coincide on a $\sigma$-finite essentially finite $W^{*}$-algebra $M$ if and only if the identity in $M$ has finite length (and a fortiori, only if $M$ is finite).

Proof. Suppose these topologies coincide on $M$. Choose a sequence of vectors $\left\{x_{n}\right\}$ such that the projections $E_{\left[M^{\prime} x_{n}\right]}$ are mutually orthogonal, $\sum_{n} E_{\left[M^{\prime} x_{n}\right]}=I$, and $\sum_{n}\left\|x_{n}\right\|^{2}<\infty$. By assumption, there exist vectors $y_{1}, \cdots, y_{n}$ such that $N_{1} \subseteq N_{2}$, where $N_{1}=\left[A \in M \mid \sum_{j=1}^{n}\left\|A y_{j}\right\|^{2}<1\right]$ and $N_{2}=\left[A \in M \mid \sum_{n}\left\|A x_{n}\right\|^{2}<1\right]$. Setting $P=\bigvee_{j} E_{\left[M^{\prime} y_{j}\right]}$, we have $K(I-P) \in N_{1}$ $\subseteq N_{2}$, for each positive real number $K$. This is possible only if $P=I$. Therefore, the identity $I$ is the union of a finite number of cyclic projections and, in turn, this implies that $I$ has finite length and that $M$ is finite.

On the other hand, suppose that the identity has finite length, and consider any sequence of vectors $\left\{x_{n}\right\}$ such that $\sum_{n}\left\|x_{n}\right\|^{2}<\infty$. By (5.6), there exist vectors $y_{1}, \cdots, y_{n}$ such that $\sum_{n} \omega_{x_{n}}=\sum_{i=1}^{n} \omega_{y_{i}}(M)$. Plainly, 
any strongest neighborhood determined by the $x_{n}$ coincides with a strong neighborhood determined by the $y_{i}$, and it follows that the strongest and the strong topologies coincide on $M$.

By a *-isomorphism between two *-algebras $M_{1}$ and $M_{2}$ we mean an algebraic isomorphism $\phi$ of $M_{1}$ with $M_{2}$ which preserves adjoints, in the sense that $\phi\left(A^{*}\right)=(\phi(A))^{*}$, for each $A$ in $M_{1}$. Such an isomorphism is called countably additive if, whenever $\left\{P_{n}\right\}$ is a sequence of mutually orthogonal projections in $M_{1}$, then $\sum_{n} P_{n}$ is in $M_{1}$ if and only if $\sum_{n} \phi\left(P_{n}\right)$ is in $M_{2}$, and then $\phi\left(\sum_{n} P_{n}\right)=\sum_{n} \phi\left(P_{n}\right)$. It is straightforward to verify that any *-isomorphism between $W^{*}$-algebras is countably additive.

Corollary 5.3. Any *-isomorphism between $\sigma$-finite finite $W^{*}$-algebras is bicontinuous in the strongest topologies. If the identities in each of these $W^{*}$ algebras have finite length, then this *-isomorphism is weakly bicontinuous.

Proof. We shall consider only the second assertion, the proof in the general case being altogether similar. Let $M_{1}$ and $M_{2}$ be $\sigma$-finite finite $W^{*}$-algebras on hilbert spaces $\mathfrak{S}_{1}$ and $\mathfrak{S}_{2}$, both $M_{1}$ and $M_{2}$ having identity of finite length, and let $\phi$ be a ${ }^{*}$-isomorphism of $M_{1}$ on $M_{2}$. Our second assertion will plainly follow if we prove that $\phi$ is weakly continuous at 0 , in $M_{1}$. Accordingly, we shall prove that if $N_{2}$ is a (general) weak neighborhood of 0 in $M_{2}$, then there exists a weak neighborhood $N_{1}$ of 0 in $M_{1}$ such that $\phi\left(N_{1}\right) \subseteq N_{2}$.

Let $N_{2}=\left[A\right.$ in $M_{2}||\left(A x_{i}, y_{i}\right) \mid<\epsilon$, for $\left.i=1, \cdots, n\right]$. As follows easily by polarization, it will suffice to consider the case when $x_{i}=y_{i}$, for all $i$. Define for each $i$ a CA state $\rho_{i}$ of $M_{1}$ by the equation $\rho_{i}(A)=\omega_{x_{i}}(\phi(A)), A$ in $M_{1}$. Applying (5.6), we choose vectors $z_{1, i}, \cdots, z_{n_{i}, i}$ in $\mathfrak{S}_{1}$ such that $\rho_{i}$ $=\sum_{j=1}^{n_{i}} \omega_{z_{j, i}}(M)$. Let $K=\max _{i} n_{i}$, and define $N_{1}=\left[A\right.$ in $M_{1}||\left(A z_{i, j}, z_{i, j}\right) \mid$ $<\epsilon / K$, for all $i$ and $j]$. It is easily verified that $N_{2} \supseteq \phi\left(N_{1}\right)$.

The hypothesis of $\sigma$-finiteness in the first assertion of this corollary is not really necessary, as follows from the fact that a *-isomorphism between $W^{*}$-algebras is necessarily completely additive, in conjunction with (3) of remark 5.1. In fact, any completely additive ${ }^{*}$-isomorphism of a finite $W^{*}$ algebra in a $W^{*}$-algebra is (relatively) strongest continuous.

We prove now what is by all rights the strongest of the topological corollaries of Theorem 4 -that, under appropriate $\sigma$-finiteness conditions, a CA *-isomorphic image of a finite $W^{*}$-algebra is itself a (finite) $W^{*}$-algebra. For related results, see von Neumann [11] and Dixmier [2].

CoROLlARY 5.4. Let $\phi$ be a countably additive *-isomorphism of a $\sigma$-finite finite $W^{*}$-algebra $M$ on a hilbert space $\mathfrak{W}$ in a $\sigma$-finite $W^{*}$-algebra $M_{0}$ on a hilbert space $\mathfrak{W}_{0}$. Suppose that the image $\phi(M)$ of $M$ in $M_{0}$ is dense in $M_{0}$ in the weak neighborhood topology. Then the image $\phi(M)$ coincides with $M_{0}$.

Proof. Since a countable central direct sum of weakly closed rings is itself weakly closed, we see, by appealing to the decomposition afforded by 
Theorem 3, that it will suffice to assume the identity in $M$ has finite length. This done, we shall prove that the closure of $\phi(M)$ in the strongest topology coincides with $M_{0}$. Since the strongest closure of a *algebra coincides with its weak closure (see e.g. [2, Theorem 8]), this will establish our assertion.

Let $T_{0}$ be an element (in $M_{0}$, necessarily) belonging to the strongest closure of $\phi(M)$. By the $\sigma$-finiteness of $M_{0}$, we can choose a sequence $\left\{x_{r}\right\}$ of vectors in $\mathfrak{S}_{0}$ such that $\sum_{r}\left\|x_{r}\right\|^{2}<\infty$, the subspaces [ $\left.M_{0}^{\prime} x_{r}\right]$ are mutually orthogonal, and $\mathfrak{S}_{0}=\sum_{r} \oplus\left[M_{0}^{\prime} x_{r}\right]$. Since by definition each strongest neighborhood of $T_{0}$ contains an element of $\phi(M)$, there exists for each $n$ an element $A_{n}$ in $M$ such that $\sum_{r}\left\|\left(\phi\left(A_{n}\right)-T_{0}\right) x_{r}\right\|<1 / n$. Let $\rho$ be that state of $M$ defined by $\rho(A)=\sum_{r} \omega_{x_{r}}(\phi(A)), A$ in $M$. Since $\phi$ is CA, it is easily seen that $\rho$ is CA. Moreover, it follows easily from our definition and the algebraic properties of $\phi$ that $\lim _{n, m} \rho\left(\left(A_{n}-A_{m}\right)^{*}\left(A_{n}-A_{m}\right)\right)=0$. Now, according to (5.6), there exist vectors $\xi_{1}, \cdots, \xi_{N}$ in $\mathfrak{S}$ such that $\rho=\sum_{i=1}^{N} \omega_{\xi_{i}}(M)$. In addition, since $L_{2}(M, \rho)$ is complete (by (5.9)), there exists a $T$ in $L_{2}(M, \rho)$ such that $\lim _{n} \sum_{i=1}^{N}\left\|\left(A_{n}-T\right) \xi_{i}\right\|=0$. We shall devote the remainder of the proof to showing that $T \in M$ and $\phi(T)=T_{0}$.

Using the polar decomposition, we write $T=U H$, where $U$ is a unitary operator in $M$ and $H$ is a non-negative SA element in $L_{2}(M, \lambda)$; recall also that this decomposition is unique. Let $E(\lambda)$ be the spectral family of $H$. Since the mapping $\phi$ is continuous in the strong sequential topology (as follows from the remark after Corollary 5.3), it is easy to see that $E_{0}(\lambda)=\phi(E(\lambda))$ is a spectral family on $\mathfrak{S}_{0}$. Let $H_{0}$ be the unique non-negative SA operator on $\mathfrak{S}_{0}$ having $E_{0}(\lambda)$ as its spectral family, and define $\phi(T)=\phi(U) H_{0}$. It is clear that $\phi(T) \in C\left(M_{0}\right)$. Define $H_{n}=E(n) H$ and $\left(H_{0}\right)_{n}=E_{0}(n) H_{0}$. For each $n, H_{n}$ and $\left(H_{0}\right)_{n}$ are bounded non-negative SA operators. Moreover, by the continuity of $\phi$ and the spectral theorem, $\left(H_{0}\right)_{n}=\phi\left(H_{n}\right)$, and in addition the following criterion holds: a vector $\xi$ in $\mathfrak{S}$ (or a vector $x$ in $\mathfrak{W}_{0}$ ) belongs to Domain $H$ (or Domain $H_{0}$ ) if and only if $\lim _{n}\left\|H_{n} x\right\|^{2}$ exists (or $\lim _{n}\left\|\left(H_{0}\right)_{n} x\right\|^{2}$ exists), and then $H \xi=\lim _{n} H_{n} \xi$ (or $H_{0} x=\lim _{n}\left(H_{0}\right)_{n} x$ ).

Applying our definitions, we have $\sum_{r}\left\|\left(H_{0}\right)_{n} x_{r}\right\|^{2}=\sum_{r} \omega_{x_{r}}\left(H_{n}^{2}\right)$ $=\sum_{i=1}^{N}\left\|H_{n} \xi_{i}\right\|^{2}$, for all $n$. But the $\xi_{i} \in$ Domain $H$, since $H \in L_{2}(M, \rho)$, and therefore $\lim _{n} \sum_{i=1}^{N}\left\|H_{n} \xi_{i}\right\|^{2}$ exists. It results that each vector $x_{r}$ belongs to Domain $H_{0}$. In addition, $\lim _{n} A_{n} \xi_{i}=\lim _{n} U H_{n} \xi_{i}=U H \xi_{i}=T \xi_{i}$, for each $i$. Therefore, $\lim _{n} \rho\left(\left(A_{n}-U H_{n}\right)^{*}\left(A_{n}-U H_{n}\right)\right)=0$. Therefore, $\phi(T) x_{r}=\phi(U) H_{0} x_{r}$ $=\lim _{n} \phi(U) \phi\left(H_{n}\right) x_{r}=\lim _{n} \phi\left(A_{n}\right) x_{r}=T_{0} x_{r}$, for each $r$. The operator $T_{0}$ is by definition bounded, and the operator $\phi(T)$ is closed. Moreover, $T_{0}=\phi(T)$ everywhere on a dense manifold in $\mathfrak{S}_{0}$-in fact, on all finite sums $\sum_{i} B_{i} x_{i}$, where the $B_{i} \in\left(M_{0}\right)^{\prime}$. Therefore, $T_{0}=\phi(T)$ everywhere on $\mathfrak{S}_{0}$, and the operator $\phi(T)$ is bounded. Notice then that $\phi(T)$ is the strong sequential limit of the $\phi\left(U H_{n}\right)$. We shall complete the proof by showing that $H$ (and therefore $T$ ) is itself bounded.

In view of the fact that an everywhere defined SA operator is bounded, 
it will suffice to prove that $H$ is everywhere defined. This will result if we prove that, for each vector $\xi$ in $\mathfrak{S}, \lim _{n}\left\|H_{n} \xi\right\|^{2}$ exists. Now, $\phi(M)$ is a $C^{*}$-algebra (see [14], also [4]). We define on $\phi(M)$ a positive functional $\tau$ by the equation $\tau(\phi(A))=\omega_{\xi}(A)(A$ in $M)$, and in these terms it will suffice to prove that the numbers $\tau\left(\phi\left(H_{n}^{2}\right)\right)$ are uniformly bounded. But $\tau$ is necessarily (uniformly) continuous on $\phi(M)$, and since the operators $\phi\left(H_{n}\right)$ tend to $\phi(H)$ in the strong sequential topology, the $\phi\left(H_{n}\right)$ are uniformly bounded. Putting these facts together, the assertion follows.

REMARK 5.2. Let $M$ be a factor of type $\mathrm{II}_{1}$ (on a separable hilbert space, by definition). We shall show that no CA state of $M$ can be pure, in the sense, e.g., of [13]. To this end, construct (as in (5.6) or [13]) the cyclic *-representation $\phi_{\rho}$ of $M$ on $\mathfrak{S}_{\rho}$, where $\rho$ is a given CA state of $M$. As is well known, the state $\rho$ is pure if and only if the ${ }^{*}$-algebra $\phi_{\rho}(M)$ is irreducible. Suppose that $\rho$ is pure, then. The representation $\phi_{\rho}$ will have kernel 0 , since $M$ contains no nontrivial uniformly closed 2-sided ideals, and it is easily seen that this *-isomorphism is countably additive. By using the fact that $M$ contains a strong-sequentially dense (uniformly) separable $C^{*}$-subalgebra (see Segal [16]), it follows readily that $\mathfrak{S}_{\rho}$ is separable. The $\sigma$-finiteness conditions of Corollary 5.4 are satisfied, and we see that $\phi_{\rho}(M)$ is a $W^{*}$-algebra. By our assumption, $\phi_{\rho}(M)$ must therefore coincide with the ring of all bounded linear operators on $\mathfrak{S}_{\rho}$. But this is impossible, since $M$ is a $\mathrm{II}_{1}$, and it follows that $\rho$ cannot be pure.

6. Associated $H$-systems. Consider a fixed CA unitarily invariant state $\lambda$ of the $\sigma$-finite finite $W^{*}$-algebra $M$, the identity of which has finite length. We shall analyze the structure of $L_{2}(M, \lambda)$ in some detail, drawing from this analysis the conclusion that $L_{2}(M, \lambda)$ can in a natural way be assigned the structure of an $H$-system (see Ambrose [1]) whose bounded algebra contains an identity for multiplication. In Theorem 5 , we then show that any $\sigma$-finite finite $W^{*}$-algebra is ${ }^{*}$-isomorphic to the $W^{*}$-algebra $\&$ of left multiplications by elements of the bounded algebra in some finite $H$-system, and that this representation of $M$ by $\&$ enjoys the uniqueness properties described in the introduction. Incidentally, the fact that the algebra $\&$ on any finite $H$-system is necessarily $\sigma$-finite affords a natural motive for using $\sigma$-finiteness hypotheses. Developments along the lines of this section are made by Murray and von Neumann for factors in the pairing $\left(\mathrm{II}_{1}, \mathrm{II}_{1}, \alpha=1\right)$ in [9] and by von Neumann (for infinite factors) in [10, especially Theorem V].

In the following series of propositions ((6.1)-(6.10)), we denote by $\lambda$ a fixed unitarily invariant CA state of the $\sigma$-finite finite $W^{*}$-algebra $M$, and we assume that the carrier projection $E_{\lambda}=I$. Moreover, we shall require throughout that the identity in $M$ have finite length. The hilbert space $L_{2}(M, \lambda)$ with inner product $[X, Y]$ is defined as in $\S 5$. We shall make the following convention about products of elements in $C(M)$ : if $S, T \in C(M)$, then $S \circ T$ will denote the ordinary operator product of $S$ and $T$, whereas $S T$ will de- 
note the unique closed extension of $S \circ T$ in $C(M)$ (which exists by (5.3)).

We define an adjoint operation $X \rightarrow X^{*}$ on $L_{2}(M, \lambda)$ as follows: if $X$ $\in L_{2}(M, \lambda)$, then $X^{*}$ is the (operator) adjoint of $X$ (as an element of $C(M)$ ).

(6.1) If $X \in L_{2}(M, \lambda)$, then $X^{*} \in L_{2}(M, \lambda)$, and $[X, X]=\left[X^{*}, X^{*}\right]$.

For elements $A$ in $M$, the relation $[A, A]=\left[A^{*}, A^{*}\right]$ is an obvious consequence of the unitary invariance of $\lambda$. For a general $X$ in $L_{2}(M, \lambda)$, the result follows by a now familiar approximation argument (based on the existence of a sequence $\left\{X_{n}\right\}$ in $M$ such that $[X, X]=\lim _{n}\left[X_{n}, X_{n}\right]$ and $\left[X^{*}, X^{*}\right]$ $\left.=\lim _{n}\left[X_{n}^{*}, X_{n}^{*}\right]\right)$.

With each $X$ in $L_{2}(M, \lambda)$ we associate an operator $L_{X}$ on $L_{2}(M, \lambda)$ defined as follows: let $z_{1}, \cdots, z_{n}$ be any finite set of vectors such that $\lambda$ $=\sum_{i=1}^{n} \omega_{z_{i}}(M)$; then an element $Y$ of $L_{2}(M, \lambda)$ belongs to Domain $L_{X}$ if the vectors $Y z_{1}, \cdots, Y z_{n} \in$ Domain $X$, and then $L_{X} Y=X Y$. (Notice that this requirement is stronger than the condition $X Y \in L_{2}(M, \lambda)$ alone.) In view of (5.5), we see that this definition does not depend on the particular $z_{i}$ chosen to represent $\lambda$ in canonical form.

(6.2) For each $X$ in $L_{2}(M, \lambda), L_{X}$ is a closed operator on $L_{2}(M, \lambda)$ with domain containing $M$ (viewed as a dense subspace of $L_{2}(M, \lambda)$ ).

By (6.1) and definition, $A^{*} X^{*} \in L_{2}(M, \lambda)$ for each $A$ in $M$. Also $\left(A^{*} X^{*}\right)^{*}$ $=\left(A^{*} \circ X^{*}\right)^{*}=X A \in L_{2}(M, \lambda)$, by (6.1) and (5.3). Since $A^{*}$ is bounded, $\left(A^{*} \circ X^{*}\right)^{*}=X \circ A$. Therefore, by definition, $A \in$ Domain $L_{X}$.

To conclude, we show that $L_{X}$ is closed-if $\left\{Y_{m}\right\}$ is a sequence in $L_{2}(M, \lambda)$ belonging to Domain $L_{X}$, if $\lim _{m} Y_{m}=Y \in L_{2}(M, \lambda)$, and if $\left\{L_{X} Y_{m}\right\}$ is cauchy, then $Y \in$ Domain $L_{X}$ and $X Y=\lim _{m} X Y_{m}$. Write $\lambda=\sum_{i=1}^{n} \omega_{z_{i}}(M)$. Since $\left[L_{X}\left(Y_{m}-Y_{p}\right), L_{X}\left(Y_{m}-Y_{p}\right)\right]=\sum_{i=1}^{n}\left\|X\left(Y_{m} z_{i}-Y_{p} z_{i}\right)\right\|^{2}$ tends to 0 with $m$ and $p$, and since $X$ is closed, it follows that $Y z_{i} \in$ Domain $X$ and $(X Y) z_{i}=X\left(Y z_{i}\right)$ $=\lim _{m} X\left(Y_{m} z_{i}\right)=\lim _{m}\left(X Y_{m}\right) z_{i}$, for each $i$. That is, $X Y=\lim _{m} X Y_{m}$ in $L_{2}(M, \lambda)$.

(6.3) The operator $L_{A}\left(A\right.$ in $\left.L_{2}(M, \lambda)\right)$ is everywhere defined if and only if $A \in M$.

If $A \in M$, it is obvious that $L_{A}$ is everywhere defined. Conversely, suppose that $L_{A}$ is everywhere defined. By definition, therefore, if $\lambda=\sum_{i=1}^{n} \omega_{z_{i}}(M)$ is any representation of $\lambda$, each subspace $\left[M z_{i}\right] \subseteq$ Domain $A$. Let $y$ be any vector in $\mathfrak{S}$. By Corollary 5.1 , there exist vectors $z_{1}, \cdots, z_{n}$ such that $\lambda=\sum_{i=1}^{n} \omega_{z_{i}}(M)$ and $y \in\left[M^{\prime} z_{1}\right]$. Since $y$ is therefore integrable $\left(M^{\prime}, z_{1}\right)$, we can find a partial isometry $V^{\prime}$ in $M^{\prime}$ such that $\left(V^{\prime}\right)^{*} V^{\prime} y=y$ and $V^{\prime} y \in\left[M z_{1}\right]$ (see Lemma 3.1). By what we have proved, $\left[M z_{1}\right] \subseteq$ Domain $A$. Therefore, $V^{\prime} y \in$ Domain $A$, and also $\left(V^{\prime}\right)^{*} V^{\prime} y=y \in$ Domain $A$. Therefore $A$ is everywhere defined on the hilbert space $\mathfrak{S}$. Since a closed everywhere defined operator is bounded, $A \in M$.

Let $A_{l}(M, \lambda)$ denote the collection of all $L_{A}\left(A\right.$ in $\left.L_{2}(M, \lambda)\right)$ which are everywhere defined on $L_{2}(M, \lambda)$ (or, what comes to the same thing, which are bounded). It is plain that $\mathcal{A}_{l}(M, \lambda)$ is a *algebra of operators (with 
identity) on $L_{2}(M, \lambda)$.

(6.4) For $A$ in $M$, the mapping $A \rightarrow L_{A}$ is $a^{*}$-isomorphism of $M$ onto $A_{l}(M, \lambda)$. 1].

This is proved in a much more general context by Segal in $[13$, Theorem

Before proceeding further with the study of left multiplications, it will be convenient to introduce a notion of right multiplication by elements of $L_{2}(M, \lambda)$. With each $X$ in $L_{2}(M, \lambda)$ we associate a (linear) operator $R_{X}$ on $L_{2}(M, \lambda)$ defined as follows: for any $Y$ in $L_{2}(M, \lambda), R_{X} Y$ is defined if and only if $L_{Y} X$ is defined, and then $R_{X} Y=L_{Y} X$.

In particular, if $A \in M$, then $R_{A}$ is everywhere defined by (6.2). Moreover $R_{A}$ is a closed operator (viz. bounded), for if $X_{n} \rightarrow 0$, then $\lim _{n}\left[R_{A} X_{n}, R_{A} X_{n}\right]$ $=\lim _{n}\left[L_{A} * X_{n}^{*}, L_{A} * X_{n}^{*}\right]=0$, using (6.1) and the fact that $L_{A} *$ is bounded. This collection of operators $R_{A}(A$ in $M)$ forms a ${ }^{*}$-algebra of operators with identity; for all $A, B$ in $M$ and all scalars $\alpha, \beta$, one has $R_{(\alpha A+\beta B)}=\alpha R_{A}+\beta R_{B}$, $R_{B A}=R_{A} R_{B},\left(R_{A}^{*}\right)^{*}=R_{A}$, and $R_{I}=$ the identity operator on $L_{2}(M, \lambda)$, as one verifies by the method cited in (6.4), making additional use of the unitary invariance of $\lambda$.

(6.5) $A_{l}(M, \lambda)$ is the commutant of the *algebra of operators $R_{A}(A$ in $M)$. As such, $A_{l}(M, \lambda)$ is a $\sigma$-finite finite $W^{*}$-algebra of operators on $L_{2}(M, \lambda)$.

Let $\bar{T}$ be any bounded operator on $L_{2}(M, \lambda)$ commuting with all the $R_{A}$. In particular, we have $\bar{T} R_{A} I=R_{A} \bar{T} I$, and if we write $\bar{T}(I)=X$, this becomes $\bar{T}(A)=X A=L_{X} A$, by (6.2). Therefore, $L_{X}=\bar{T}$ densely, and since $L_{X}$ is closed, $L_{X}=\bar{T}$ everywhere. So by (6.3), $X \in M$, and by definition, $\bar{T}$ $\in A_{l}(M, \lambda)$. Since the commutant of any *algebra of operators is a $W^{*}$ algebra, $\mathcal{A}_{l}(M, \lambda)$ is necessarily a $W^{*}$-algebra. And since $\mathcal{A}_{l}(M, \lambda)$ is *-isomorphic with the $\sigma$-finite finite $W^{*}$-algebra $M$, by $(6.4), A_{l}(M, \lambda)$ is itself a $\sigma$-finite finite $W^{*}$-algebra. (Of course, the latter fact follows also by Corollary 5.4.)

(6.6) For any elements $X$ and $Y$ in $L_{2}(M, \lambda), X \in$ Domain $L_{Y}$ if and only if $Y^{*} \in$ Domain $L_{X^{*}}$.

We shall use the following fact: given any two elements $S, T$ in $C(M)$, there exists a sequence $\left\{R_{m}\right\}$ of projections in $M$ with the following properties: the operators $S R_{m}$ and $T R_{m}$ are bounded for each $m$, and a vector $x$ belongs to Domain $S$ (or Domain $T$ ) if and only if $\lim _{m}\left(S R_{m}\right) x$ exists (or $\lim _{m}\left(T R_{m}\right) x$ exists). The existence of such a sequence was established in the uniqueness proof of Corollary 5.1.

In the present circumstances, writing $\lambda=\sum_{i=1}^{n} \omega_{z_{i}}(M)$, we know by definition that the $z_{i} \in$ Domain $X, X z_{i} \in$ Domain $Y$, and by (6.1) that the $z_{i}$ $\in$ Domain $Y^{*}$, and we are to prove that the $Y^{*} z_{i} \in$ Domain $X^{*}$. Choose a sequence $\left\{R_{m}\right\}$ of the sort described above for the operators $Y$ and $X^{*}$. We must then show that $\lim _{p, m} \sum_{i=1}^{n}\left\|\left(X^{*} R_{p}-X^{*} R_{m}\right)\left(Y^{*} z_{i}\right)\right\|^{2}=0$. Now $\sum_{i=1}^{n}\left\|\left(X^{*} R_{p}-X^{*} R_{m}\right)\left(Y^{*} z_{i}\right)\right\|^{2}=\sum_{i=1}^{n}\left\|\left(X^{*} R_{p} Y^{*}-X^{*} R_{m} Y^{*}\right) z_{i}\right\| \|^{2}=($ by (6.1)) 
$\sum_{i-1}^{n}\left\|\left(Y R_{p} X-Y R_{m} X\right) z_{i}\right\|^{2}=\sum_{i=1}^{n}\left\|\left(Y R_{p}-Y R_{m}\right)\left(X z_{i}\right)\right\|^{2}$, which tends to 0 with $m, p$ since $X z_{i} \in$ Domain $Y$. This proves the proposition.

(6.7) For each $X$ in $L_{2}(M, \lambda), L_{X} \in C\left(\mathcal{A}_{l}(M, \lambda)\right)$.

In view of (6.5) and a criterion cited earlier, it will suffice to prove that if $X \in L_{2}(M, \lambda)$ and if $A \in M$, then $L_{X} R_{A} \supseteq R_{A} L_{X}$ - that is, for each $Y$ in Domain $L_{X}$, it is true that $Y A \in$ Domain $L_{X}$ and that $X(Y A)=(X Y) A$.

Now, if $Y \in$ Domain $L_{X}$, then $X^{*} \in$ Domain $L_{Y}$, by (6.6). In the familiar fashion, choose a sequence $\left\{Y_{m}\right\}$ in $M$ such that $Y_{m}^{*} x \rightarrow Y^{*} x$, for each $x$ in Domain $Y^{*}$. By (6.1), $\lim _{m} Y_{m}=Y$ in $L_{2}(M, \lambda)$. Moreover, $\lim _{m} X Y_{m}=X Y$ in $L_{2}(M, \lambda)$, because $\lim _{m}\left[X Y_{m}-X Y, X Y_{m}-X Y\right]=\lim _{m}\left[\left(Y_{m}^{*}-Y^{*}\right) X^{*}\right.$, $\left.\left(Y_{m}^{*}-Y^{*}\right) X^{*}\right]=\lim _{m} \sum_{i=1}^{n}\left\|\left(Y_{m}^{*}-Y^{*}\right)\left(X^{*} z_{i}\right)\right\|^{2}=0$, by our choice of the $Y_{m}^{*}$. Now $\lim _{m} Y_{m} A=R_{A}\left(\lim _{m} Y_{m}\right)=Y A$, and also the sequence $\left\{L_{X}\left(Y_{m} A\right)\right\}$ is cauchy -in fact, since the $\left\{Y_{m}\right\}$ are bounded, $\lim _{m} L_{X}\left(Y_{m} A\right)=\lim _{m} X\left(Y_{m} A\right)$ $=\lim _{m}\left(X Y_{m}\right) A=R_{A}\left(\lim _{m} X Y_{m}\right)=(X Y) A-$ and because $L_{X}$ is a closed operator, we conclude that $Y A \in$ Domain $L_{X}, X(Y A)=\lim _{m} L_{X}\left(Y_{m} A\right)$ $=(X Y) A$, completing the demonstration.

We are now in a position to prove that the representation $X \rightarrow L_{X}$ of $L_{2}(M, \lambda)$ by left multiplications on itself preserves adjoints. For each $X$ in $L_{2}(M, \lambda)$, we denote by $l_{X}$ the contraction of the operator $L_{X}$ to the domain $M$, viewed as a dense subspace of $L_{2}(M, \lambda)$.

(6.8) For each $X$ in $L_{2}(M, \lambda), L_{X} \cdot=\left(L_{X}\right)^{*}=\left(l_{X}\right)^{*}$.

By definition, $\left(l_{X}\right)^{*} \supseteq\left(L_{X}\right)^{*}$. To prove equality, we must show that $\left[L_{X} Y, W\right]=\left[Y,\left(l_{X}\right)^{*} W\right]$, for any $Y$ in Domain $L_{X}$ and $W$ in Domain $\left(l_{X}\right)^{*}$. This follows immediately if we choose (as in (6.7)) a sequence $\left\{Y_{m}\right\}$ in $M$ such that both $\lim _{m} Y_{m}=Y$ and $\lim _{m} X Y_{m}=X Y$ in $L_{2}(M, \lambda)$ and observe that already $\left[L_{X} Y_{m}, W\right]=\left[Y_{m},\left(l_{X}\right)^{*} W\right]$, for all $m$.

By known properties of $C\left(\mathcal{A}_{l}(M, \lambda)\right)$ (e.g. (5.2)), $\left(l_{X}\right)^{*}$ and $L_{X}$. have a common dense domain in $L_{2}(M, \lambda)$. Suppose that $Y$ belongs to the common dense domain, and suppose $\lambda=\sum_{i=1}^{n} \omega_{z_{i}}(M)$. Then $\left[A, L_{X} \cdot Y\right]$ $=\sum_{i=1}^{n}\left(A z_{i}, \quad X^{*}\left(Y z_{i}\right)\right)=($ by $\quad(6.2)) \quad \sum_{i=1}^{n}\left(X\left(A z_{i}\right), \quad Y z_{i}\right)=\left[\begin{array}{ll}L_{X} A, & Y\end{array}\right]$ $=\left[A,\left(l_{X}\right)^{*} Y\right]$. Therefore, $L_{X}=\left(l_{X}\right)^{*}$ densely. But by (5.1), any two elements of $C(M)$ ( $M$ finite) which are equal densely have the same domain and coincide everywhere on this domain. Thus, $L_{X^{*}}=\left(l_{X}\right)^{*}$.

It is now easy to carry out the above program for right multiplications.

(6.9) Propositions (6.2), (6.3), (6.5), and (6.7) obtain for right multiplications and the corresponding algebra $\mathcal{A}_{r}(M, \lambda)$.

First, $R_{X}$ is closed, for each $X$ in $L_{2}(M, \lambda)$; if the sequence $\left\{Y_{m}\right\}$ $\subseteq$ Domain $R_{X}$, if $Y_{m} \rightarrow Y$, and if $\left\{R_{X} Y_{m}\right\}$ is cauchy, then $Y \in$ Domain $R_{X}$ and $\lim _{m} R_{X} Y_{m}=Y X$ in $L_{2}(M, \lambda)$. In fact, by definition, $X \in$ Domain $L_{Y_{n}}$, and by (6.6), $\left\{Y_{n}^{*}\right\} \subseteq$ Domain $L_{X}$. By (6.1), the sequence $\left\{X^{*} Y_{n}^{*}\right\}$ is cauchy and $Y_{n}^{*} \rightarrow Y^{*}$. Therefore, since $L_{X}$ is closed, $Y^{*} \in$ Domain $L_{X} \cdot$ and $X^{*} Y_{n}^{*} \rightarrow X^{*} Y^{*}$. Again by (6.6), $X \in$ Domain $L_{Y}$, so $Y \in$ Domain $R_{X}$, and by (6.1), $Y_{n} X \rightarrow Y X$.

If $R_{A}$ is everywhere defined, then $T^{*} \in$ Domain $L_{A}$, for all $T$ by definition 
and (6.6). So by (6.3), $A^{*}$ (and hence $A$ ) $\in M$. On the other hand, (6.2) shows that $R_{A}$ is everywhere defined if $A \in M$.

By the same argument given in (6.5), we see that $A_{r}(M, \lambda)$ is actually all of $\left(\mathcal{A}_{l}(M, \lambda)\right)^{\prime} . \mathcal{A}_{r}(M, \lambda)$ is then a finite $W^{*}$-algebra, being an essentially finite $W^{*}$-algebra whose commutant has a cyclic vector (viz. $I$ ). Finally, (6.7) follows for right multiplications by a simple application of (6.6) and (6.7), proving the assertions.

Denote by $r_{X}$ the contraction of $R_{X}$ to the dense manifold $M$ in $L_{2}(M, \lambda)$.

(6.10) For each $X$ in $L_{2}(M, \lambda), R_{X} \cdot=\left(R_{X}\right)^{*}=\left(r_{X}\right)$.

We show first that $\left(r_{X}\right)^{*} \in C\left(\mathcal{A}_{r}(M, \lambda)\right)$. That is, if $Y \in$ Domain $\left(r_{X}\right)^{*}$, then $(A Y) \in$ Domain $\left(r_{X}\right)^{*}$ and $\left(r_{X}\right)^{*}(A Y)=A\left(\left(r_{X}\right)^{*} Y\right)$. It being obvious that $r_{X} L_{A}=L_{A} r_{X}$ on $M$, for each $A$ in $M$, we have $\left[r_{X} B, A Y\right]$ $\left.=\left[L_{A} \cdot\left(r_{X} B\right), Y\right]=\left[r_{X}\left(A^{*} B\right), Y\right]=\left[A^{*} B\right),\left(r_{X}\right)^{*} Y\right]=\left[B, A\left(\left(r_{X}\right)^{*} Y\right)\right]$, for all $A, B$ in $M$, proving the assertion. By (5.1), therefore, $\left(R_{X}\right)^{*}=\left(r_{X}\right)^{*}$.

Notice that $[X, Y]=\left[Y^{*}, X^{*}\right]$, for all $X$ and $Y$ in $L_{2}(M, \lambda)$ [1, Lemma 2.1]. Now, to see that $\left(r_{X}\right)^{*}=R_{X}$, , consider a $Y$ in the dense manifold (Domain $\left.R_{X} \cdot\right) \cap\left(\right.$ Domain $\left.\left(r_{X}\right)^{*}\right)$ and compute $\left[A, R_{X} \cdot Y\right]=\left[A, L_{Y} X^{*}\right]$ $=($ by $(6.8))\left[L_{Y} \cdot A, X^{*}\right]=\left(\right.$ since $\left(R_{A}\right)^{*}=R_{A} \cdot$ if $\left.A \in M\right)\left[Y^{*}, X^{*} A^{*}\right]=[A X, Y]$ $=\left[\left(r_{X}\right) A, Y\right]=\left[A,\left(r_{X}\right)^{*} Y\right]$, for all $A$ in $M$. Therefore, $\left(r_{X}\right)^{*}=R_{X} \cdot$ densely. The conclusion follows as in (6.8).

We shall prove that the system at which we have arrived in (6.1)-(6.10) is really independent of the particular unitarily invariant CA state $\lambda$, up to a strong notion of equivalence. The following proposition provides the basis for this proof.

(6.11) Let $\phi_{1}$ and $\phi_{2}$ be two cyclic representations of $M$ on hilbert spaces $\mathfrak{S}_{1}$ and $\mathfrak{S}_{2}$. If $\xi_{i} \in \mathfrak{S}_{i}$ are cyclic vectors for $\phi_{i}(M)$, assume that each state (of $M$ ) $\lambda_{i}(A)=\left(\phi_{i}(A) \xi_{i}, \xi_{i}\right)$ is $C A$, unitarily invariant, and has $E_{\lambda_{i}}=I$. Then these representations $\phi_{1}$ and $\phi_{2}$ are unitarily equivalent (that is, there exists a unitary mapping $U$ of $\mathfrak{S}_{1}$ on $\mathfrak{S}_{2}$ such that $U \phi_{1}(A)=\phi_{2}(A) U$, for all $A$ in $\left.M\right)$.

First, consider any sequence $\left\{A_{n}\right\}$ in $M$ with the properties that $\lim _{n} \lambda_{1}\left(A_{n}^{*} A_{n}\right)=0$ and $\lim _{n, m} \lambda_{2}\left(\left(A_{n}-A_{m}\right)^{*}\left(A_{n}-A_{m}\right)\right)=0$. We shall show that $\lim _{n} \lambda_{2}\left(A_{n}^{*} A_{n}\right)=0$ also. In fact, by Corollary 5.1 , we may write $\lambda_{1}$ $=\sum_{i=1}^{n} \omega_{x_{i}}(M)$ and $\lambda_{2}=\sum_{i=1}^{n} \omega_{y_{i}}(M)$, with the vectors $x_{1}, \cdots, x_{n}$ and $y_{1}, \cdots, y_{n}$ so chosen that $E_{\left[M^{\prime} x_{i}\right]}=E_{\left[M^{\prime} y_{i}\right]}$, for each $i$. By hypothesis, $\lim _{n} A_{n} x_{i}=0$ and $\left\{A_{n} y_{i}\right\}$ is cauchy, for each $i$. But $y_{i}$ is integrable $\left(M^{\prime}, x_{i}\right)$ for each $i$, by Theorem 2 , and this implies that $\lim _{n} A_{n} y_{i}=0$. The assertion follows and, by symmetry, the same condition holds when the roles of the $\lambda_{i}$ are reversed.

Next, we define a linear operator $T_{0}$ from $\mathfrak{S}_{1}$ to $\mathfrak{S}_{2}$ as follows: $T_{0}\left(\phi_{1}(A) \xi_{1}\right)$ $=\phi_{2}(A) \xi_{2}$, for each $A$ in $M$. Plainly, $T_{0}$ is well defined, linear, with dense range and domain, and furthermore $T_{0} \phi_{1}(A)=\phi_{2}(A) T_{0}$ on $\phi_{1}(M) \xi_{1}$. We shall show that $T_{0}$ has a closed nonsingular extension $T$ (a fortiori, with dense range and domain), with the property that $T \phi_{1}(A) \supseteq \phi_{2}(A) T$, for all $A$ in $M$. 
Considering the graph of $T_{0}$, it is easily seen that $T_{0}$ has a closed extension if and only if $\lim _{n} \phi_{1}\left(A_{n}\right) \xi_{1}=0$ and $\left\{\phi_{2}\left(A_{n}\right) \xi_{2}\right\}$ cauchy together imply $\lim _{n} \phi_{2}\left(A_{n}\right) \xi_{2}=0$; and furthermore, that if this condition is realized, then the operator $T$ whose graph is the closure of the graph of $T_{0}$ will be nonsingular if and only if $\lim _{n} \phi_{2}\left(A_{n}\right) \xi_{2}=0$ and $\left\{\phi_{1}\left(A_{n}\right) \xi_{1}\right\}$ cauchy together imply $\lim _{n} \phi_{1}\left(A_{n}\right) \xi_{1}=0$. In terms of the states $\lambda_{i}$, these necessary and sufficient conditions are precisely those conditions verified in the first paragraph. Therefore, $T$ exists, and from the fact that $T$ is closed and $T \phi_{1}(A)=\phi_{2}(A) T$ on $\phi_{1}(M) \xi_{1}$, it follows easily that $\phi_{2}(A) T \subseteq T \phi_{1}(A)$, for all $A$ in $M$.

But now, according to the generalized Schur lemma proved by Neumark in $[12, \S 7]$, the representations $\phi_{1}$ and $\phi_{2}$ are unitarily equivalent; in effect, if one applies the polar decomposition to $T$ to write $T=U\left(T^{*} T\right)^{1 / 2}$, the ascribed properties of $T$ imply that $U$ is a unitary mapping of $\mathfrak{S}_{1}$ on $\mathfrak{S}_{2}$ with the property that $U \phi_{1}(A)=\phi_{2}(A) U$, for all $A$ in $M$. This proves (6.11).

We recall the definition of an $H$-system (Ambrose [1, p. 28]). An $H$-system $H$ is a complex hilbert space in which a partial multiplication is defined and satisfies the following conditions: (i) the collection $A$ of elements in $H$ which right and left multiply every element of $H$ is a dense manifold and an algebra under operations in $H$; (ii) $x A=0$ implies $x=0$, for any $x$ in $H$; (iii) there is associated with each element $x$ of $H$ an element $x^{*}$ (called the adjoint of $x$ ) satisfying $\|x\|=\left\|x^{*}\right\|$ and giving rise to the following operator equalities: $L_{x^{*}}=\left(L_{x}\right)^{*}=\left(l_{x}\right)^{*}$ and $R_{x^{*}}=\left(R_{x}\right)^{*}=\left(r_{x}\right)^{*}$, where $L_{x}: y \rightarrow x y$ (and $R_{x}: y \rightarrow y x$ ) is defined for all $y$ such that $x y$ (or $y x$ ) is defined, and where $l_{x}$ (or $r_{x}$ ) is the contraction of $L_{x}$ (or $R_{x}$ ) to the manifold $A$. Two $H$-systems $H_{1}$ and $H_{2}$ are called isomorphic if there exists a mapping of $H_{1}$ on $H_{2}$ which preserves sums, scalar multiples, products when defined, adjoints, and norms. Finally, we call an $H$-system finite if the bounded algebra $A$ of $H$ contains a multiplicative identity $e$.

(6.12) The collection \& of all left multiplications $L_{a}$ by elements of the bounded algebra $A$ in a finite $H$-system forms a $\sigma$-finite finite $W^{*}$-algebra in the natural operations. If $e$ denotes the identity in $A$, then $\omega_{\theta}$ is a $C A$ unitarily invariant state of $\mathfrak{R}$, and $H=L_{2}\left(\mathfrak{R}, \omega_{e}\right), \mathfrak{R}=\mathcal{A}_{l}\left(\mathfrak{R}, \omega_{e}\right)$. Finally, the commutant $\mathfrak{R}^{\prime}$ of $\mathfrak{R}$ coincides with the collection $\Re$ of all right multiplications $R_{a}$ by elements of the bounded algebra $A$.

(6.13) Two finite $\mathrm{H}$-systems $\mathrm{H}_{1}$ and $\mathrm{H}_{2}$ are isomorphic (as $\mathrm{H}$-systems) if and only if the corresponding $W^{*}$-algebras $\mathfrak{R}_{1}$ and $\mathfrak{R}_{2}$ of bounded left multiplications are unitarily equivalent.

The proof of (6.12) is elementary (see e.g. (6.5)) and is omitted. Regarding (6.13), it is obvious that an $H$-system isomorphism of $H_{1}$ and $H_{2}$ induces a unitary equivalence of $R_{1}$ and $\Omega_{2}$. Conversely, suppose that $U$ is a unitary operator from $H_{1}$ on $H_{2}$ with the property that $U \Omega_{1}=\Omega_{2} U$. From the fact that identity elements must correspond we see easily that, for each $L_{a}$ in $\Omega_{1}$, we have $U L_{a}=L_{U a} U$. It results that the mapping $a \rightarrow U a$ from the bounded 
algebra $A_{1}$ of $H_{1}$ in the bounded algebra $A_{2}$ of $H_{2}$ is a *-isomorphism onto; for example, $L_{U a^{*}}=U L_{a} \cdot U^{*}=\left(U L_{a} U^{*}\right)^{*}=\left(L_{U a}\right)^{*}=L_{(U a)}$, so $U a^{*}=(U a)^{*}$, and $L_{U a b}=\left(U L_{a} U^{*}\right)\left(U L_{b} U^{*}\right)=L_{U a} L_{U b}$, so $U(a b)=(U a)(U b)$. Now, by means of the familiar approximation argument (see (6.7)), one proves also that $U L_{x}=L_{U x} U$, for all $x$ in $H_{1}$, and using this fact, it follows in a straightforward fashion that $x \rightarrow U x$ is actually an $H$-system isomorphism.

THEOREM 5. Given a $\sigma$-finite finite $W^{*}$-algebra $M$, there exists $a *^{*}$-isomorphism $A \rightarrow L_{A}$ between $M$ and the $W^{*}$-algebra $\&$ of left multiplications by elements of the bounded algebra of a finite $H$-system $H(M)$. This associated finite $H$-system $H(M)$ is determined uniquely up to $H$-system isomorphisms by the existence of this *-isomorphism $A \rightarrow L_{A}$. Furthermore, two $\sigma$-finite finite $W^{*}$ algebras $M_{1}$ and $M_{2}$ are ${ }^{*}$-isomorphic if and only if the associated finite $H$-systems $H\left(M_{1}\right)$ and $H\left(M_{2}\right)$ are isomorphic as $H$-systems, or what is the same, if and only if the corresponding algebras $\mathfrak{R}_{1}$ and $\mathfrak{R}_{2}$ of bounded left multiplications are unitarily equivalent.

Proof. To begin, we shall proceed with the assumption that the identity in $M$ has finite length. The existence of $H(M)$ is assured by the construction given in (6.1)-(6.10). Corresponding to a fixed unitarily invariant CA state $\lambda$ of $M$ with $E_{\lambda}=I$, the underlying hilbert space of $H(M)$ is $L_{2}(M, \lambda)$, and the partial multiplication is that obtained by defining the product $X Y$ of two elements $X$ and $Y$ in $L_{2}(M, \lambda)$ to be $L_{X} Y\left(=R_{Y} X\right)$, this being defined if and only if $Y \in$ Domain $L_{X}$.

Let $H_{0}$ be any other finite $H$-system, with the property that $M$ is *-isomorphic to the $W^{*}$-algebra $\Omega_{0}$ of bounded left multiplications in $H_{0}$, and let $e_{0}$ be the identity in $H_{0}$. Then $\lambda_{0}(A)=\omega_{e_{0}}\left(L_{A}\right)$ is a CA unitarily invariant state of $M$, with $E_{\lambda_{0}}=I$. According to (6.11), the representation algebras $\mathfrak{R}_{0}$ and $\mathfrak{R}$ on $H_{0}$ and $H(M)$ are unitarily equivalent. Therefore, by (6.13), $H_{0}$ is isomorphic to $H(M)$. By the same token, two finite $W^{*}$-algebras whose identities have finite length are *-isomorphic if and only if their associated $H$-systems are isomorphic.

Turning to the general case, we shall show first that any $\sigma$-finite finite $W^{*}$-algebra $M$ is ${ }^{*}$-isomorphic to a finite $W^{*}$-algebra whose identity has length 1 . In fact, using Theorem 3 , express $M$ as a central direct sum of finite $W^{*}$-algebras $M_{n}$ uniformly of length $n$. Now, by the case treated above, each $M_{n}$ is ${ }^{*}$-isomorphic to a finite $W^{*}$-algebra $\bar{M}_{n}$ whose identity has length 1 . Since a countable direct sum of finite $W^{*}$-algebras of length 1 is easily seen to be a finite $W^{*}$-algebra of length 1 , we conclude that $M$ is *-isomorphic to $\bar{M}=\sum_{n} \bar{M}_{n}$, a finite $W^{*}$-algebra of length 1 .

We now define $H(M)$, for an arbitrary $\sigma$-finite finite $W^{*}$-algebra $M$, to be the finite $H$-system associated with any finite $W^{*}$-algebra which is *-isomorphic to $M$ and whose identity has finite length. By the first case, this definition is invariant; and the systems $H(M)$ obviously fulfill the require- 
ments of the theorem.

Corollary 5.4 shows that the *-isomorphism $A \rightarrow L_{A}$ will be strongly continuous when $M$ has finite length; on the other hand, if $M$ has infinite length, then it can be shown easily that this isomorphism cannot be strongly continuous.

A (stronger) abelian analogue of Theorem 5 is proved by Segal in $[18$, Theorem 4.1]. For analogues of (6.5) and (6.12) in the case of $L_{2}$-systems of discrete groups, see von Neumann [11], or for general unimodular LC groups, Segal [15].

\section{REFERENCES $(1)$}

1. W. Ambrose, The $L_{2}$-system of a unimodular group. I, Trans. Amer. Math. Soc. vol. 65 (1949) pp. 27-48.

2. J. Dixmier, Les fonctionnelles linéaires sur l'ensemble des opérateurs bornés d'un espace de Hilbert, Ann. of Math. vol. 51 (1950) pp. 387-408.

3. - Les anneaux d'opérateurs de classe fini, Ann. Ecole Norm (3) vol. 66 (1949) pp. 209-261.

4. I. Gelfand and M. Neumark, On the imbedding of normed rings into the ring of operators in Hilbert space, Rec. Math. (Mat. Sbornik) N.S. vol. 12 (1943) pp. 197-213.

5. I. Kaplansky, Quelques résultats sur les anneaux d'opérateurs, C. R. Acad. Sci. Paris, vol. 123 (1950) pp. 485-486.

6. - - Projections in Banach algebras, Ann. of Math. vol. 53 (1951) pp. 235-249.

7. F. Maeda, Relative dimensionality in operator rings, Journal of Science of Hirosima University vol. 11 (1941) pp. 1-6.

8. F. Murray and J. von Neumann, On rings of operators, Ann. of Math. vol. 37 (1936) pp. 116-229.

9. - On rings of operators. II, Trans. Amer. Math. Soc. vol. 41 (1937) pp. 208-248.

10. J. von Neumann, On rings of operators. III, Ann. of Math. vol. 41 (1940) pp. 94-161.

11. - On rings of operators. IV, Ann. of Math. vol. 44 (1943) pp. 716-808.

12. M. Neumark, Rings with involutions (in Russian), Uspehi Matematičeskih Nauk N.S. vol. 3 (1948) pp. 52-145.

13. I. Segal, Irreducible representations of operator algebras, Bull. Amer. Math. Soc. vol. 53 (1947) pp. 73-88. 865.

14. - , Two-sided ideals in operator algebras, Ann. of Math. vol. 50 (1949) pp. 856-

15. - The two-sided regular representation of a unimodular locally compact group, Ann. of Math. vol. 51 (1950) pp. 293-298.

9.

16. - Decomposition theory. I, Memoirs of the American Mathematical Society, no.

17. - Decomposition theory. II, Memoirs of the American Mathematical Society, no. 9.

18. - Equivalences of measure spaces, Amer. J. Math. vol. 73 (1951) pp. 275-313.

California Institute of Technology, Pasadena, Calif.

(1) For an exposition of the spectral theory, see B. v. Sz. Nagy, Spektraldarstellung linearer Transformationen des Hilbertschen Raumes, Berlin, 1942. An extensive bibliography on rings of operators is found in [8]. 